\title{
Nonlinear Unknown Input Observer Based on Singular Value Decomposition Aided Reduced Dimension Cubature Kalman Filter
}

\author{
Wei Zhao, ${ }^{1,2}$ Huiguang Li, ${ }^{1}$ Liying Zou, ${ }^{1}$ and Wenjuan Huang ${ }^{3}$ \\ ${ }^{1}$ School of Electrical Engineering, Yanshan University, No. 438, Hebei Street, Qinhuangdao 066004, China \\ ${ }^{2}$ Qinggong College, North China University of Science and Technology, No. 11, Daxue West Road, Tangshan 063000, China \\ ${ }^{3}$ Tangshan Vocational and Technical College, No. 120, Xinhua West Road, Tangshan 063004, China
}

Correspondence should be addressed to Wei Zhao; zhwei19800@163.com

Received 19 September 2016; Accepted 18 January 2017; Published 7 March 2017

Academic Editor: Alberto Borboni

Copyright (C) 2017 Wei Zhao et al. This is an open access article distributed under the Creative Commons Attribution License, which permits unrestricted use, distribution, and reproduction in any medium, provided the original work is properly cited.

The paper presents a nonlinear unknown input observer (NUIO) based on singular value decomposition aided reduced dimension Cubature Kalman filter (SVDRDCKF) for a special class of nonlinear systems, the nonlinearity of which is only caused by part of its states. Firstly, the algorithm of general NUIO is discussed and the unknown input observer based on singular value decomposition aided Cubature Kalman filter (SVDCKF) given. Then a special nonlinear system model with unknown input is introduced. Based on the proposed model and the corresponding NUIO, the equivalent integral form with partial sampling and all sampling of the state vector in Cubature Kalman filter is analyzed. Finally the nonlinear unknown input observer based on singular value decomposition aided reduced dimension Cubature Kalman filter is obtained. Simulation results show that the proposed algorithm can meet the requirements of the system and is more important to increase the calculating efficiency a lot, although it has a decline in the accuracy of the filter.

\section{Introduction}

The optimal control theory is able to improve the characteristics of the system. In most instances, control theory often needs to estimate the state variables and parameters in practical application. However, the actual control system has the uncertainty, which makes the robust control theory develop rapidly and be one of the most active research fields. The Kalman filter [1] proposed in 1960s is an optimal linear minimum variance estimation method. The Kalman filter has strict requirements on the system model, and the uncertainty of the model will make the performance of the filter drop or even diverge. Sayed [2] divides the robust Kalman filter method into four kinds, (i) $H$ - $\infty$ filtering, (ii) set-valued estimation, (iii) guaranteed-cost filtering, and (iv) regularized least-squares.

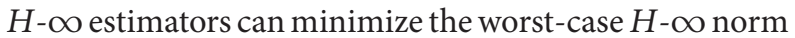
of the power spectral density or keep it the prescribed constraints [3]. Set-valued estimation recursively computes the bounded ellipsoid corresponding to sets of possible states using observations assuming that the collection of initial condition, input noise, and observation noise can be approximated as ellipsoid [4]. The method of guaranteedcost filtering designs an upper bound of variation of the estimated error for the reasonable system, and the resultant estimator minimizes the upper bound [5]. The regularized least-squares filter presented by Sayed [2] minimizes the worst-possible regularized residual norm over the class of admissible uncertainties at each iteration.

In recent years, the unknown input observer (UIO), as a robust estimator, is gradually becoming the focus in the robust control field [6]. The observer treats the model uncertainty and external disturbance as the unknown input of the system and can eliminate their influence on the system by decoupling the unknown inputs. The UIO has been widely used in aerospace [7] and chemical industry [8].

For nonlinear systems, the early presented EKF [9] can do nothing for the complex nonlinear systems, especially 
when the nonlinear system cannot be linearized by Taylor's expansion [10]. To improve the performances of Kalman filter, the algorithms, such as Unscented Kalman Filter (UKF) [11], Cubature Kalman filter (CKF) [12], and Particle Filter (PF) [13], are proposed to deal with nonlinear system. Then these algorithms are combined with NUIO for the state estimation, fault diagnosis and detection. The algorithm of linear UIO model is extended to nonlinear UIO system, and gets better applications. For example, in literature [8] the linear UIO model is expanded to NUIO to estimate the gain of the observer in which Unscented Transformation (UT) is used. Among the aforementioned filters, the CKF algorithm with its excellent performance, such as higher stability and accuracy as well as faster convergence rate than those of UKF, has been widely used in the high dimensional nonlinear system [14].

For linear systems, the reduced order or full order UIO has been given in many literatures. The literature [15] proposes a filtering method for unknown input using system identification. Based on the reduced order UIO, the robust detection problem of intermittent failures for a class of linear discrete time stochastic systems with time-varying parameter disturbances and unknown disturbances is studied in [16].

At present, in the NUIO system, the solution to reduce the dimensionality of the problem is mainly concentrated in reduction of dimension of UIO combining with the nonlinearities of the system [17]; on the contrary, considering the nonlinearity of the system, the paper uses the reduced dimension algorithm to design the full order NUIO, whose purpose is to improve the computational efficiency. As known that using the UKF or CKF in the NUIO system it is needed to sample the state, which is termed sampling point. The UKF requires selecting $2 n+1$ sampling points for the state, while CKF needs $2 n$ sampling points. When the number of system state is small, the influence of the number of sampling points on the computational efficiency is not obvious. However, when the number is more than a few, this effect may be revealed [18].

In this paper, a nonlinear unknown input observer based on singular value decomposition aided reduced dimension Cubature Kalman Filter (NUIO based on SVDRDCKF) is discussed. The greatest contribution of this paper is reducing the number of sampling points to improve the computational efficiency of NUIO for a special class of nonlinear systems. In practice, it is known that, for some nonlinear systems, its nonlinearity is not caused by all the elements of the state vector. It may be that only part of the system states makes the system nonlinear, so if the CKF only samples the state vector resulting nonlinearly, it will significantly reduce the number of sampling points. The smaller number of sampling points means higher computational efficiency. In this paper, the equivalent integral form used in CKF for nonlinear propagation between the nonlinear part and all of state vector is given. Although the reduction of the sampling points will make the estimated accuracy slightly lower than that of the conventional CKF, it does not affect the accuracy of the CKF to the third-order Taylor's expansion.

The organization of the remaining part is as follows: Section 2 presents the preliminary knowledge about NUIO that how the general form of NUIO is obtained. The complete algorithm of NUIO based on SVDCKF is given in Section 3. In Section 4, we firstly give a special nonlinear NUIO model, based on which the theorem for the equivalent integral form of the part and all sampling points is proved. Then the NUIO based on SVDRDCKF algorithm is obtained consequently. Analysis is made in Section 5 to justify the performances of the proposed algorithm compared with the NUIO based on SVDCKF algorithm through the simulation of the maneuvering target tracking system. Finally, the conclusion is given in Section 6.

\section{Preliminary Knowledge about NUIO}

There are usually two different ways to process the unknown input in the existing methods. The first method [19] relies on the introduction of an additional matrix into the state estimation equation, which is used to decouple the effects of unknown inputs on the state estimation error. The second one [20] is to transform the system with the unknown input into a system without unknown input. In this paper, we will take second methods as the theoretical basis for the design of NUIO.

Consider a nonlinear time-variant discrete time stochastic system

$$
\begin{aligned}
& x_{k+1}=f\left(x_{k}, u_{k}\right)+E_{k} d_{k}+w_{k} \\
& y_{k+1}=h\left(x_{k+1}\right)+v_{k},
\end{aligned}
$$

where $x$ is the $n$-dimensional system state vector, $y$ is the $m$ dimensional measurement vector, and $d$ is the $q$-dimensional unknown input (or disturbance) with its corresponding distribution matrix $E$. $f(\cdot)$ and $h(\cdot)$ are the nonlinear equations. $w \sim N\left(0, Q_{w}\right)$ and $v \sim N\left(0, Q_{v}\right)$ represent the $n$-dimensional process noise and $m$-dimensional measurement noise, respectively.

Without loss of generality, the measurement equation is generally linearized as follows:

$$
C_{k+1}=\frac{\partial h\left(x_{k+1}\right)}{\partial x_{k+1}}
$$

where $C_{k+1}$ is the linearized measurement matrix and $\partial h\left(x_{k+1}\right) / \partial x_{k+1}$ is the partial derivative of function $h\left(x_{k+1}\right)$ on $x_{k+1}$.

Then the measurement equation is obtained as

$$
y_{k+1}=C_{k+1} x_{k+1}+v_{k} .
$$

The necessary condition for the existence of a solution to the unknown input decoupling problem is $\operatorname{rank}\left(C_{k+1} E_{k}\right)=$ $\operatorname{rank}\left(E_{k}\right)$. If the condition is satisfied, then it is possible to calculate the parameter matrix $H$ of the observer [21].

$$
\begin{aligned}
H_{k+1} & =\left(C_{k+1} E_{k+1}\right)^{+} \\
& =\left[\left(C_{k+1} E_{k+1}\right)^{T} C_{k+1} E_{k+1}\right]^{-1}\left(C_{k+1} E_{k+1}\right)^{T} .
\end{aligned}
$$

Note 1. The symbol "+" represents the pseudo inverse of matrix. $H_{k+1}$ is the solution of (12). 
Multiply (5) by (4) and substitute (1) into the resultant equation. Then we have

$$
\begin{aligned}
H_{k+1} y_{k+1}= & H_{k+1} C_{k+1} x_{k+1}+H_{k+1} v_{k} \\
= & H_{k+1} C_{k+1}\left[f\left(x_{k}, u_{k}\right)+E_{k} d_{k}+w_{k}\right] \\
& +H_{k+1} v_{k} .
\end{aligned}
$$

Retaining only the term $E_{k} d_{k}$ on the right of (6) with the other moved to left, (6) can be received as

$$
\begin{aligned}
& H_{k+1}\left[y_{k+1}-C_{k+1}\left(f\left(x_{k}, u_{k}\right)+w_{k}\right)-v_{k}\right] \\
& \quad=H_{k+1} C_{k+1} E_{k} d_{k}=\left(C_{k+1} E_{k+1}\right)^{+} C_{k+1} E_{k} d_{k}=d_{k} .
\end{aligned}
$$

Substitute (7) into (1).

$$
\begin{aligned}
x_{k+1}= & f\left(x_{k}, u_{k}\right)+E_{k} d_{k}+w_{k} \\
= & f\left(x_{k}, u_{k}\right) \\
& +E_{k} H_{k+1}\left[y_{k+1}-C_{k+1}\left(f\left(x_{k}, u_{k}\right)+w_{k}\right)-v_{k}\right] \\
& +w_{k}=f\left(x_{k}, u_{k}\right)+E_{k} d_{k}+w_{k} \\
= & f\left(x_{k}, u_{k}\right) \\
& +E_{k} H_{k+1}\left[y_{k+1}-C_{k+1}\left(f\left(x_{k}, u_{k}\right)+w_{k}\right)\right] \\
& -E_{k} H_{k+1} v_{k}+w_{k} \\
= & \left(I-E_{k} H_{k+1} C_{k+1}\right) f\left(x_{k}, u_{k}\right)+E_{k} H_{k+1} y_{k+1} \\
& +\left(I-E_{k} H_{k+1} C_{k+1}\right) w_{k}-E_{k} H_{k+1} v_{k} \\
= & \bar{f}\left(x_{k}, u_{k}\right)+\bar{E}_{k} y_{k+1}+\left(I-E_{k} H_{k+1} C_{k+1}\right) w_{k} \\
& -E_{k} H_{k+1} v_{k} .
\end{aligned}
$$

Then the UIO for system represented by (1) and (4) can be shown as follows:

$$
\begin{aligned}
\widehat{x}_{k+1 / k} & =\bar{f}\left(\widehat{x}_{k}, u_{k}\right)+\bar{E}_{k} y_{k+1} \\
\widehat{x}_{k+1} & =\widehat{x}_{k+1 / k}+K_{k+1}\left(y_{k+1}-\widehat{y}_{k+1 / k}\right),
\end{aligned}
$$

where $\bar{f}\left(\widehat{x}_{k}, u_{k}\right)=\left(I-E_{k} H_{k+1} C_{k+1}\right) f\left(\widehat{x}_{k}, u_{k}\right)$ and $\bar{E}_{k}=$ $E_{k} H_{k+1}$. The observer gain $K_{k}$ can be obtained using the Kalman filter algorithm, which will be discussed in Section 3 in detail.

Note 2. The symbol “-” above a variable represents its estimated value.

Necessary and sufficient conditions [21] for the UIO in (9) and (10) of the defined system in (1) and (2) are as follows:

(1)

$$
\operatorname{rank}\left(C_{k+1} E_{k}\right)=\operatorname{rank}\left(E_{k}\right)
$$

(2)

$$
E_{k}=H_{k+1} C_{k+1} E_{k}
$$

(3) $K_{k+1}$ should make the observer stable.

\section{NUIO Based on SVDCKF}

According to the obtained UIO in (9) and (10), the observer gain $K_{k}$ can be obtained under the CKF framework [22]. As the covariance matrix may lose positive definite in abnormal condition while using the Cholesky decomposition in CKF, it can be used to substitute the singular value decomposition of matrix for the corresponding ones. In such way, the stability of the numerical calculation can be improved. Combined with the literature $[7,22]$, this paper gives the complete algorithm of NUIO based on SVDCKF as follows.

\section{(A) Initialization}

(1) Initialize with $\widehat{x}_{0}=E\left(x_{0}\right), \widehat{P}_{x, 0}=E\left(\left(x_{0}-\widehat{x}_{0}\right)\left(x_{0}-\right.\right.$ $\left.\widehat{x}_{0}\right)^{T}$ ), and $H_{0}=0$.

(2) Use (6) to calculate $H_{k+1}$ for $k=0,1,2, \ldots$, and then check the necessary and sufficient conditions for the UIO.

(B) Time Update $(k=0,1,2, \ldots)$

(1) Obtain the cubature points by singular value decomposition for $\widehat{P}_{x, k}$.

$$
\begin{aligned}
\widehat{P}_{x, k} & =U_{k}\left[\begin{array}{cc}
S_{k}^{*} & 0 \\
0 & 0
\end{array}\right] V_{k}^{T} \\
\widehat{X}_{i, k} & =U_{k} \sqrt{S_{k}^{*}} \xi_{i}+\widehat{x}_{k} \quad i=1,2, \ldots, 2 n \\
\xi_{i} & =\sqrt{n}[1]_{i},
\end{aligned}
$$

where $[1]_{i}$ is the $i$ th column of the points set [1]

(2) These cubature points are propagated through the nonlinear state equation shown in (9).

$$
\widehat{X}_{i, k+1 / k}=\bar{f}\left(\widehat{X}_{i, k}, u_{k}\right)+\bar{E}_{k} y_{k+1} \text {. }
$$

(3) Calculate the value of state prediction $\widehat{x}_{k+1 / k}$, and covariance matrix $\widehat{P}_{x, k+1 / k}$.

$\widehat{x}_{k+1 / k}=\frac{1}{2 n} \sum_{i=1}^{2 n} \widehat{X}_{i, k+1 / k}$.

$\widehat{P}_{x, k+1 / k}$

$$
\begin{aligned}
= & \frac{1}{2 n} \sum_{i=1}^{2 n} \widehat{X}_{i, k+1 / k} \widehat{X}_{i, k+1 / k}^{T}-\widehat{x}_{k+1 / k} \widehat{x}_{k+1 / k}^{T} \\
& +\left(I-E_{k} H_{k+1} C_{k+1}\right) Q_{k}\left(I-E_{k} H_{k+1} C_{k+1}\right)^{T} \\
& -E_{k} H_{k+1} R_{k}\left(E_{k} H_{k+1}\right)^{T}
\end{aligned}
$$

\section{(C) Measurement Update}

(1) Obtain the new cubature points by singular value decomposition for $\widehat{P}_{x, k+1 / k}$. 


$$
\begin{aligned}
& \widehat{P}_{x, k+1 / k}=U_{k}\left[\begin{array}{cc}
S_{k}^{*} & 0 \\
0 & 0
\end{array}\right] V_{k}^{T} \\
& \widehat{X}_{i, k+1 / k}^{*}=U_{k} \sqrt{S_{k}^{*}} \xi_{i}+\widehat{x}_{k+1 / k} \quad i=1,2, \ldots, 2 n .
\end{aligned}
$$

(2) These cubature points are propagated through the measurement equation shown in (4).

$$
\widehat{Y}_{i, k+1 / k}=C_{k+1} \widehat{X}_{i, k+1 / k}^{*}
$$

(3) Calculate the measurement estimation.

$$
\widehat{y}_{k+1 / k}=\frac{1}{2 n} \sum_{i=1}^{2 n} \widehat{Y}_{i, k+1 / k} \text {. }
$$

(4) Calculate the measurement prediction $\widehat{P}_{y, k+1 / k}$ and cross-covariance matrix $\widehat{P}_{x y, k+1 / k}$.

$$
\begin{aligned}
\widehat{P}_{y, k+1 / k}= & \frac{1}{2 n} \sum_{i=1}^{2 n} \widehat{Y}_{i, k+1 / k}\left(\widehat{Y}_{i, k+1 / k}\right)^{T} \\
& -\widehat{y}_{k+1 / k}\left(\widehat{y}_{k+1 / k}\right)^{T}+R_{k} \\
\widehat{P}_{x y, k+1 / k}= & \frac{1}{2 n} \sum_{i=1}^{2 n} \widehat{X}_{i, k+1 / k}\left(\widehat{Y}_{i, k+1 / k}\right)^{T} \\
& -\widehat{x}_{k+1 / k}\left(\widehat{y}_{k+1 / k}\right)^{T} .
\end{aligned}
$$

(D) State Update

(1) The observer gain $K_{k+1}$ can be obtained as

$$
K_{K+1}=\widehat{P}_{x y, k+1 / k} \widehat{P}_{y y, k+1 / k}
$$

(2) Calculate the state estimation.

$$
\widehat{x}_{k+1}=\widehat{x}_{k+1 / k}+K_{k+1}\left(y_{k+1}-\widehat{y}_{k+1 / k}\right) .
$$

(3) Calculate covariance matrix.

$$
\widehat{P}_{x, k+1}=\widehat{P}_{x, k+1 / k}-K_{k+1} \widehat{P}_{y y, k+1 / k} K_{k+1}^{T} .
$$

\section{NUIO Based on SVDRDCKF}

4.1. System Model with Special Nonlinearity. For some special nonlinear models, the cause that makes KF unable to be applied to the system is found to be that only some elements of the system state make the whole system nonlinear. The system model to be studied below belongs to this case. Before using the reduced dimension algorithm for the design of NUIO, the system expressed by (1) needs to be reformed.

Assumption 1. Assume that the UIO system described by (1) can be transformed into a special nonlinear systems shown as (26).

$$
\begin{aligned}
& x_{k+1}=F\left(\zeta_{k}, u_{k}\right) x_{k}+g\left(\zeta_{k}, u_{k}\right)+E_{k} d_{k}+w_{k} \\
& y_{k+1}=C_{k+1} x_{k+1}+v_{k},
\end{aligned}
$$

where $\zeta$ is first $l$ elements (linear part) of $x$.
If $\eta$ is defined as the last $n-l$ elements (nonlinear part) of $x$, then $x=\left[\begin{array}{ll}\zeta^{T} & \eta^{T}\end{array}\right]^{T}$.

According to the analysis in Section 2, the NUIO for system in (26) can be obtained as follows:

$$
\begin{aligned}
\widehat{x}_{k+1 / k} & =\bar{f}\left(\widehat{x}_{k}, u_{k}\right)+\bar{E}_{k} y_{k+1} \\
= & \left(I-E_{k} H_{k+1} C_{k+1}\right) f\left(x_{k}, u_{k}\right)+E_{k} H_{k+1} y_{k+1} \\
= & \left(I-E_{k} H_{k+1} C_{k+1}\right)\left(F\left(\zeta_{k}, u_{k}\right) x_{k}+g\left(\zeta_{k}, u_{k}\right)\right) \\
& +E_{k} H_{k+1} y_{k+1} . \\
\widehat{x}_{k+1}= & \widehat{x}_{k+1 / k}+K_{k+1}\left(y_{k+1}-\widehat{y}_{k+1 / k}\right)
\end{aligned}
$$

In order to understand and identify more easily, the nonlinear function in (27) is defined in Definition 2 for the SVDCKF.

Definition 2. For the nonlinear system described by (27), define that, at instant $k(k=0,1,2, \ldots), Z=(I-$ $\left.E_{k} H_{k+1} C_{k+1}\right)\left(F\left(\zeta_{k}, u_{k}\right) x_{k}+g\left(\zeta_{k}, u_{k}\right)\right)+E_{k} H_{k+1} y_{k+1}$, where $x_{k} \sim N\left(x_{k} ; \widehat{x}_{k}, P_{x, k}\right)$ with $n$ dimension. $\zeta_{k}$ is first $l$ elements (linear part) of $x_{k} \cdot y_{k+1}, E_{k}, H_{k+1}, C_{k+1}$, and $u_{k}$ are known vector or matrix aforementioned.

4.2. Relative Theorems. According to the NUIO given by (27) and (28), it needs to select the $2 n$ sampling points if NUIO based on SVDCKF is used to obtain the observer gain $K_{k}$ (See (14)). Due to that only partial elements of the system state are nonlinear, if we only sample on the nonlinear part of state rather than on the entire state, it can reduce the number of sampling points so as to the amount of the filtering computation. This resultant filter to be discussed is termed as singular value decomposition aided reduced dimension Cubature Kalman filter (SVDRDCKF).

In order to obtain a priori estimates of the state with the same third-order accuracy using reduced dimension algorithm compared with regular algorithm, we first give the Lemma 3 [18].

Lemma 3. When $x=\left[\zeta_{1}, \zeta_{2}, \ldots, \zeta_{l}, \eta_{1}, \eta_{2}, \ldots, \eta_{n-l}\right]^{T}(0 \leq l \leq$ $n$ and $\left.x \sim N\left(x ; 0, I_{n}\right)\right)$, where $\zeta=\left[\zeta_{1}, \zeta_{2}, \ldots, \zeta_{l}\right]^{T}$ and $\eta=$ $\left[\eta_{1}, \eta_{2}, \ldots, \eta_{n-l}\right]^{T}$, the following equations should be true:

$$
\begin{aligned}
E\left(\eta_{i} f(\zeta)\right) & =0 \\
E\left(\eta_{i} \eta_{j} f(\zeta)\right) & =0 \\
E\left(\eta_{i}^{2} f(\zeta)\right) & =E(f(\zeta)) .
\end{aligned}
$$

Proof.

(A) Prove $E\left(\eta_{i} f(\zeta)\right)=0$.

$$
E\left(\eta_{i} f(\zeta)\right)=\frac{1}{(2 \pi)^{n / 2}} \int \eta_{i} f(\zeta) e^{-x^{T} x / 2} d x=\frac{1}{(2 \pi)^{n / 2}}
$$




$$
\begin{aligned}
& \cdot \int \eta_{i} e^{-\eta_{i}^{2} / 2} d \eta_{i} \cdot \int f(\zeta) e^{-\zeta \zeta^{T} / 2} d \zeta \\
& \cdot \int e^{-\left(\eta_{1}^{2}+\eta_{2}^{2}+\cdots+\eta_{i-1}^{2}+\eta_{i+1}^{2}+\cdots+\eta_{n-l}^{2}\right) / 2} d \eta_{1} d \eta_{2} \cdots d \eta_{i-1} d \eta_{i+1} \\
& \cdots d \eta_{n-l} .
\end{aligned}
$$

As $\int \eta_{i} e^{-\eta_{i}^{2} / 2} d \eta_{i}=0, E\left(\eta_{i} f(\zeta)\right)=0$, so (29) is true.

(B) Prove $E\left(\eta_{i} \eta_{j} f(\zeta)\right)=0$.

Similar to the proof of $E\left(\eta_{i} \eta_{j} f(\zeta)\right)=0$, (30) is true too.

(C) Prove $E\left(\eta_{i}^{2} f(\zeta)\right)=E(f(\zeta))$.

$$
\begin{aligned}
& E\left(\eta_{i}^{2} f(\zeta)\right)=\frac{1}{(2 \pi)^{n / 2}} \int \eta_{i}^{2} f(\zeta) e^{-x^{T} x / 2} d x=\frac{1}{(2 \pi)^{n / 2}} \\
& \quad \cdot \int \eta_{i}^{2} e^{-\eta_{i}^{2} / 2} d \eta_{i} \cdot \int f(\zeta) e^{-\zeta^{T} \zeta / 2} d \zeta \\
& \quad \cdot \int e^{-\left(\eta_{1}^{2}+\eta_{2}^{2}+\cdots+\eta_{i-1}^{2}+\eta_{i+1}^{2}+\cdots+\eta_{n-l}^{2}\right) / 2} d \eta_{1} d \eta_{2} \cdots d \eta_{i-1} d \eta_{i+1} \\
& \quad \cdots d \eta_{n-l} .
\end{aligned}
$$

As

$$
\begin{aligned}
& \int \eta_{i}^{2} e^{-\eta_{i}^{2} / 2} d \eta_{i}=(2 \pi)^{1 / 2}, \\
& \int e^{-\left(\eta_{1}^{2}+\eta_{2}^{2}+\cdots+\eta_{i-1}^{2}+\eta_{i+1}^{2}+\cdots+\eta_{n-l}^{2}\right) / 2} d \eta_{1} d \eta_{2} \cdots d \eta_{i-1} d \eta_{i+1} \\
& \quad \cdots d \eta_{n-l}=(2 \pi)^{(n-l-1) / 2}
\end{aligned}
$$

we have

$$
\begin{aligned}
E\left(\eta_{i}^{2} f(\zeta)\right) & =\frac{1}{(2 \pi)^{(n-l) / 2}} \int f(\zeta) e^{-\zeta^{T} \zeta / 2} d \zeta \\
& =E(f(\zeta)) .
\end{aligned}
$$

Thus, (31) is true.

Theorem 4. For $Z=\left(I-E_{k} H_{k+1} C_{k+1}\right)\left(F\left(\zeta_{k}, u_{k}\right) x_{k}+g\left(\zeta_{k}\right.\right.$, $\left.\left.u_{k}\right)\right)+E_{k} H_{k+1} y_{k+1}$, the equivalent forms of $\widehat{Z}=\int Z N\left(x_{k}\right.$; $\left.\widehat{x_{k}}, P_{x, k}\right) d x_{k}$ and $P_{Z}=\int Z Z^{T} N\left(x_{k} ; \widehat{x_{k}}, P_{x, k}\right) d x_{k}-\widehat{Z}(\widehat{Z})^{T}$ can be expressed as follows.

$$
\begin{aligned}
\widehat{Z} & =\int \Phi\left(\zeta_{k}\right) N\left(\zeta_{k} ; \widehat{\zeta}_{k}, P_{\zeta, k}\right) d \zeta_{k} \\
P_{Z} & =\int \Psi\left(\zeta_{k}\right) N\left(\zeta_{k} ; \widehat{\zeta}_{k}, P_{\zeta, k}\right) d \zeta_{k}-\widehat{Z}(\widehat{Z})^{T},
\end{aligned}
$$

where $P_{\zeta, k}$ is the matrix composed of elements of the former $l$ rows and former $l$ columns of $P_{x, k}$; $S$ and $S_{\zeta_{k}}$ are the Cholesky decomposition of $P_{x, k}$ and $P_{\zeta, k}$, respectively; $\bar{\zeta}_{k}$ is first l elements of $\widehat{x}_{k}$; and

$$
\begin{aligned}
& \Phi\left(\zeta_{k}\right)=\left(I-E_{k} H_{k+1} C_{k+1}\right) F\left(\zeta_{k}, u_{k}\right) \\
& \cdot\left(\widehat{x}+S\left[\begin{array}{c}
\left(S_{\zeta_{k}}\right)^{-1}\left(\zeta_{k}-\widehat{\zeta}_{k}\right) \\
0_{n-l}
\end{array}\right]\right) \\
& +\left(I-E_{k} H_{k+1} C_{k+1}\right) g\left(\zeta_{k}\right)+E_{k} H_{k+1} y_{k+1} \cdot \\
& \Psi\left(\zeta_{k}\right)=\Phi\left(\zeta_{k}\right)\left(\Phi\left(\zeta_{k}\right)\right)^{T}+\left(I-E_{k} H_{k+1} C_{k+1}\right) F\left(\zeta_{k}\right) \\
& \cdot S\left[\begin{array}{cc}
0 & 0 \\
0 & I_{n-l}
\end{array}\right] S^{T} F^{T}\left(\zeta_{k}\right)\left(I-E_{k} H_{k+1} C_{k+1}\right)^{T}
\end{aligned}
$$

Proof. Define $\eta_{k}$ as the last $n-l$ elements of $x_{k}$; then $x_{k}=$ $\left[\begin{array}{ll}\zeta_{k}^{T} & \eta_{k}^{T}\end{array}\right]^{T}$. As $x_{k} \sim N\left(x_{k} ; \widehat{x}_{k}, P_{x, k}\right)$, then $\zeta_{k} \sim N\left(\zeta_{k} ; \widehat{\zeta}_{k}, P_{\zeta, k}\right)$.

(A) Firstly, prove that $\widehat{Z}=\int \Phi^{*}\left(\zeta_{k}\right) N\left(\zeta_{k} ; \bar{\zeta}_{k}, P_{\zeta, k}\right) d \zeta_{k}$ is true.

$$
\begin{aligned}
\widehat{Z} & =E(Z) \\
& =E\left(\left(I-E_{k} H_{k+1} C_{k+1}\right)\left(F\left(\zeta_{k}, u_{k}\right) x_{k}+g\left(\zeta_{k}\right)\right)\right. \\
& \left.+E_{k} H_{k+1} y_{k+1}\right)=\left(I-E_{k} H_{k+1} C_{k+1}\right) \\
& \cdot E\left(F\left(\zeta_{k}, u_{k}\right) x_{k}\right)+\left(I-E_{k} H_{k+1} C_{k+1}\right) E\left(g\left(\zeta_{k}\right)\right) \\
& +E_{k} H_{k+1} y_{k+1} .
\end{aligned}
$$

Obviously $E\left(g\left(\zeta_{k}\right)\right)$ is the Gauss integral for $\zeta_{k}$; the next proof is that $E\left(F\left(\zeta_{k}, u_{k}\right) x_{k}\right)$ is also the Gauss integral for $\zeta_{k}$.

$$
\begin{aligned}
E & \left(F\left(\zeta_{k}, u_{k}\right) x_{k}\right) \\
& =\int F\left(\zeta_{k}, u_{k}\right)\left[\begin{array}{l}
\zeta_{k} \\
\eta_{k}
\end{array}\right] N\left(x_{k} ; \widehat{x_{k}}, P_{x, k}\right) d x_{k} .
\end{aligned}
$$

Making Cholesky decomposition for $P_{x, k}$ and $P_{\zeta, k}$, we have $P_{x, k}=S S^{T}$ and $P_{\zeta, k}=S_{\zeta_{k}}\left(S_{\zeta_{k}}\right)^{T}$. As $S$ and $S_{\zeta_{k}}$ are the lower triangular matrix, it is known that $S_{\zeta_{k}}$ is the matrix composed of elements of the former $l$ rows and former $l$ columns of $S$.

Define $x_{k}=\widehat{x}_{k}+S \widetilde{x}_{k}$, where $\widetilde{x}_{k}=\left[\begin{array}{ll}\widetilde{\zeta}_{k}^{T} & \tilde{\eta}_{k}^{T}\end{array}\right]^{T}$. As $S_{\zeta_{k}}$ is the lower triangular matrix and $\zeta_{k}=\widehat{\zeta}_{k}+S_{\zeta_{k}} \widetilde{\zeta}_{k}$, we have

$$
\begin{gathered}
\int F\left(\zeta_{k}, u_{k}\right)\left[\begin{array}{l}
\zeta_{k} \\
\eta_{k}
\end{array}\right] N\left(x_{k} ; \widehat{x}_{k}, P_{x, k}\right) d x_{k}=\frac{1}{(2 \pi)^{n / 2}} \\
\cdot \int F\left(\widehat{\zeta}_{k}+S_{\zeta_{k}} \widetilde{\zeta}_{k}, u_{k}\right)\left(\widehat{x}_{k}+S\left[\begin{array}{l}
\widetilde{\zeta}_{k} \\
\tilde{\eta}_{k}
\end{array}\right]\right) e^{-\left(\widetilde{x}_{k}\right)^{T} \widetilde{x}_{k} / 2} d \widetilde{x}_{k} . \\
\text { Defining } F_{1}\left(\widehat{\zeta}_{k}+S_{\zeta_{k}} \widetilde{\zeta}_{k}, u_{k}\right)=F\left(\widetilde{\zeta}_{k}, u_{k}\right) \text {, we have } \\
E\left(F\left(\zeta_{k}, u_{k}\right) x_{k}\right)=\frac{1}{(2 \pi)^{n / 2}} \\
\cdot \int F_{1}\left(\widehat{\zeta}_{k}+S_{\zeta_{k}} \widetilde{\zeta}_{k}, u_{k}\right) \widehat{x}_{k} e^{-\left(\tilde{x}_{k}\right)^{T} \widetilde{x}_{k} / 2} d \widetilde{x}_{k}+\frac{1}{(2 \pi)^{n / 2}} \\
\cdot \int F_{1}\left(\widehat{\zeta}_{k}+S_{\zeta_{k}} \widetilde{\zeta}_{k}, u_{k}\right) S\left[\begin{array}{l}
\widetilde{\zeta}_{k} \\
\widetilde{\eta}_{k}
\end{array}\right] e^{-\left(\tilde{x}_{k}\right)^{T} \widetilde{x}_{k} / 2} d \widetilde{x}_{k} .
\end{gathered}
$$


6

Mathematical Problems in Engineering

The first term on the right side of (43) can be written as

$$
\begin{aligned}
& \frac{1}{(2 \pi)^{n / 2}} \int F_{1}\left(\widetilde{\zeta}_{k}, u_{k}\right) \widehat{x}_{k} e^{-\left(\widetilde{x}_{k}\right)^{T} \widetilde{x}_{k} / 2} d \widetilde{x}_{k} \\
& =\frac{1}{(2 \pi)^{l / 2}} \int F_{1}\left(\widetilde{\zeta}_{k}, u_{k}\right) \widehat{x}_{k} e^{-\left(\widetilde{\zeta}_{k}\right)^{T} \widetilde{\zeta}_{k} / 2} d \widetilde{\zeta}_{k} \\
& =\int F_{1}\left(\widetilde{\zeta}_{k}, u_{k}\right) \widehat{x}_{k} N\left(\widetilde{\zeta}_{k} ; 0, I_{l}\right) d \widetilde{\zeta}_{k} .
\end{aligned}
$$

The second term on the right side of (43) can be written as

$$
\begin{aligned}
& \frac{1}{(2 \pi)^{n / 2}} \int F_{1}\left(\widetilde{\zeta}_{k}, u_{k}\right) S\left[\begin{array}{c}
\tilde{\zeta}_{k} \\
\tilde{\eta}_{k}
\end{array}\right] e^{-\left(\widetilde{x}_{k}\right)^{T} \tilde{x}_{k} / 2} d \tilde{x}_{k} \\
& =\frac{1}{(2 \pi)^{n / 2}} \int F_{1}\left(\widetilde{\zeta}_{k}, u_{k}\right) S\left[\begin{array}{c}
\widetilde{\zeta}_{k} \\
0
\end{array}\right] e^{-\left(\tilde{x}_{k}\right)^{T} \widetilde{x}_{k} / 2} d \widetilde{x}_{k} \\
& \quad+\frac{1}{(2 \pi)^{n / 2}} \int F_{1}\left(\widetilde{\zeta}_{k}, u_{k}\right) S\left[\begin{array}{c}
0 \\
\tilde{\eta}_{k}
\end{array}\right] e^{-\left(\tilde{x}_{k}\right)^{T} \widetilde{x}_{k} / 2} d \widetilde{x}_{k} .
\end{aligned}
$$

The first term on the right side of (45) can be written as

$$
\begin{aligned}
& \frac{1}{(2 \pi)^{n / 2}} \int F_{1}\left(\widetilde{\zeta}_{k}, u_{k}\right) S\left[\begin{array}{c}
\widetilde{\zeta}_{k} \\
0
\end{array}\right] e^{-\left(\tilde{x}_{k}\right)^{T} \widetilde{x}_{k} / 2} d \widetilde{x}_{k} \\
& =\frac{1}{(2 \pi)^{l / 2}} \int F_{1}\left(\widetilde{\zeta}_{k}, u_{k}\right) S\left[\begin{array}{c}
\widetilde{\zeta}_{k} \\
0
\end{array}\right] e^{-\left(\widetilde{\zeta}_{k}\right)^{T} \widetilde{\zeta}_{k} / 2} d \widetilde{\zeta}_{k} \\
& =\int F_{1}\left(\widetilde{\zeta}_{k}, u_{k}\right) S\left[\begin{array}{c}
\widetilde{\zeta}_{k} \\
0
\end{array}\right] N\left(\widetilde{\zeta}_{k} ; 0, I_{l}\right) d \widetilde{\zeta}_{k} .
\end{aligned}
$$

The second term on the right side of (45) can be written as

$$
\begin{gathered}
\frac{1}{(2 \pi)^{n / 2}} \int F_{1}\left(\widetilde{\zeta}_{k}, u_{k}\right) S\left[\begin{array}{c}
0 \\
\tilde{\eta}_{k}
\end{array}\right] e^{-\left(\tilde{x}_{k}\right)^{T} \widetilde{x}_{k} / 2} d \widetilde{x}_{k} \\
=\int F_{1}\left(\widetilde{\zeta}_{k}, u_{k}\right) S\left[\begin{array}{c}
0 \\
\tilde{\eta}_{k}
\end{array}\right] N\left(\widetilde{\zeta}_{k} ; 0, I_{n}\right) d \widetilde{\zeta}_{k} \\
=\int\left[\begin{array}{c}
\sum_{i=1}^{n-l} f_{1 i}\left(\widetilde{\zeta}_{k}, u_{k}\right) \tilde{\eta}_{k i} \\
\vdots \\
\sum_{i=1}^{n-l} f_{n i}\left(\widetilde{\zeta}_{k}, u_{k}\right) \widetilde{\eta}_{k i}
\end{array}\right] N\left(\widetilde{\zeta}_{k} ; 0, I_{n}\right) d \widetilde{\zeta}_{k} .
\end{gathered}
$$

According to Lemma 3, we have

$$
\begin{aligned}
E\left(f_{j i}\left(\widetilde{\zeta}_{k}, u_{k}\right) \tilde{\eta}_{k i}\right)= & 0 \\
& i=1,2, \ldots, n-l ; j=1,2, \ldots, n .
\end{aligned}
$$

So

$$
\begin{aligned}
& \frac{1}{(2 \pi)^{n / 2}} \int F_{1}\left(\widetilde{\zeta}_{k}, u_{k}\right) S\left[\begin{array}{c}
0 \\
\tilde{\eta}_{k}
\end{array}\right] e^{-\left(\tilde{x}_{k}\right)^{T} \tilde{x}_{k} / 2} d \widetilde{x}_{k}=0, \\
& E\left(F\left(\zeta_{k}, u_{k}\right) x_{k}\right)=\int F_{1}\left(\widetilde{\zeta}_{k}, u_{k}\right) \\
& \cdot S\left[\begin{array}{c}
\widetilde{\zeta}_{k} \\
0
\end{array}\right] N\left(\widetilde{\zeta}_{k} ; 0, I_{l}\right) d \widetilde{\zeta}_{k}=\int F\left(\zeta_{k}, u_{k}\right) \\
& \cdot\left(\widehat{x}+S\left[\left(S_{\zeta_{k}}\right)^{-1}\left(\zeta_{k}-\widehat{\zeta}_{k}\right)\right]\right) \\
& \cdot N\left(\zeta_{k} ; \widehat{\zeta}_{k}, P_{\zeta, k}\right) d \zeta_{k}
\end{aligned}
$$

As $E\left(F\left(\zeta_{k}, u_{k}\right) x_{k}\right)$ is the Gauss integral for $\zeta_{k}$, it is true that (40) is also the Gauss integral for $\zeta_{k}$. Then set

$$
\begin{aligned}
\Phi\left(\zeta_{k}\right)= & \left(I-E_{k} H_{k+1} C_{k+1}\right) F\left(\zeta_{k}, u_{k}\right) \\
& \cdot\left(\widehat{x}+S\left[\begin{array}{c}
\left.\left(S_{\zeta_{k}}\right)^{-1}\left(\zeta_{k}-\widehat{\zeta}_{k}\right)\right] \\
0
\end{array}\right]\right) \\
& +\left(I-E_{k} H_{k+1} C_{k+1}\right) g\left(\zeta_{k}\right)+E_{k} H_{k+1} y_{k+1} .
\end{aligned}
$$

Hence, (36) is proved.

(B) Secondly, Prove that $P_{Z}=\int \Psi\left(\zeta_{k}\right) N\left(\zeta_{k} ; \widehat{\zeta}_{k}, P_{\zeta, k}\right) d \zeta_{k}-$ $\widehat{Z}(\widehat{Z})^{T}$ is true.

$$
P_{Z}=E\left(Z Z^{T}\right)-\widehat{Z}(\widehat{Z})^{T} .
$$

Setting $x_{k}=\widehat{x}_{k}+S \widetilde{x}_{k}$ and as $S_{\zeta_{k}}$ is the lower triangular matrix, we have $\zeta_{k}=\widehat{\zeta}_{k}+S_{\zeta_{k}} \widetilde{\zeta}_{k}$.

Define

$$
\begin{aligned}
& \left(I-E_{k} H_{k+1} C_{k+1}\right) F\left(\widehat{\zeta}_{k}+S_{\zeta_{k}} \widetilde{\zeta}_{k}, u_{k}\right)=F_{1}\left(\widetilde{\zeta}_{k}, u_{k}\right) \\
& \left(I-E_{k} H_{k+1} C_{k+1}\right) F\left(\zeta_{k}, u_{k}\right)=F_{2}\left(\zeta_{k}, u_{k}\right) \\
& \left(I-E_{k} H_{k+1} C_{k+1}\right) g\left(\widehat{\zeta}_{k}+S_{\zeta_{k}} \widetilde{\zeta}_{k}, u_{k}\right)=g_{1}\left(\widetilde{\zeta}_{k}, u_{k}\right) \\
& \left(I-E_{k} H_{k+1} C_{k+1}\right)\left(F\left(\zeta_{k}, u_{k}\right) \widehat{x}_{k}+g\left(\zeta_{k}\right)\right)=\Gamma\left(\zeta_{k}, u_{k}\right) \\
& F_{1}\left(\widetilde{\zeta}_{k}, u_{k}\right) \widehat{x}_{k}+g_{1}\left(\widetilde{\zeta}_{k}\right)=\Gamma_{1}\left(\widetilde{\zeta}_{k}, u_{k}\right) \\
& F_{1}\left(\widetilde{\zeta}_{k}, u_{k}\right) \widehat{x}_{k}+g_{1}\left(\widetilde{\zeta}_{k}\right)+F_{1}\left(\widetilde{\zeta}_{k}, u_{k}\right) S \widetilde{x}_{k} \\
& \quad=\Lambda_{1}\left(\widetilde{\zeta}_{k}, u_{k}\right) \\
& E_{k} H_{k+1} y_{k+1}=B .
\end{aligned}
$$


Mathematical Problems in Engineering

7

$E\left(Z Z^{T}\right)$ can be represented as

$$
\begin{aligned}
E( & \left.Z Z^{T}\right) \\
& =\int\left(\left(I-E_{k} H_{k+1} C_{k+1}\right)\left(F\left(\zeta_{k}, u_{k}\right) x_{k}+g\left(\zeta_{k}\right)\right)\right. \\
& \left.+E_{k} H_{k+1} y_{k+1}\right) \\
& +\left(\left(I-E_{k} H_{k+1} C_{k+1}\right)\left(F\left(\zeta_{k}, u_{k}\right) x_{k}+g\left(\zeta_{k}\right)\right)^{T}\right. \\
& \left.+E_{k} H_{k+1} y_{k+1}\right)^{T} N\left(x_{k} ; \widehat{x}_{k}, P_{x, k}\right) d x=f_{1}\left(\tilde{x}_{k}\right) \\
& +f_{2}\left(\tilde{x}_{k}\right)+f_{2}^{T}\left(\tilde{x}_{k}\right)+f_{3}\left(\tilde{x}_{k}\right)+f_{3}^{T}\left(\tilde{x}_{k}\right)+f_{4}\left(\tilde{x}_{k}\right) \\
& +f_{5}\left(\tilde{x}_{k}\right)+f_{5}^{T}\left(\tilde{x}_{k}\right)+B B^{T},
\end{aligned}
$$

where

$$
\begin{aligned}
& f_{1}\left(\widetilde{x}_{k}\right)=\int \Gamma_{1}\left(\widetilde{\zeta}_{k}, u_{k}\right) \Gamma_{1}^{T}\left(\widetilde{\zeta}_{k}, u_{k}\right) N\left(\tilde{x}_{k} ; 0, I_{n}\right) d \widetilde{x}_{k} \\
& f_{2}\left(\tilde{x}_{k}\right)=\int \Gamma_{1}\left(\widetilde{\zeta}_{k}, u_{k}\right)\left(F_{1}\left(\widetilde{\zeta}_{k}, u_{k}\right) S \tilde{x}_{k}\right)^{T} \\
& \cdot N\left(\tilde{x}_{k} ; 0, I_{n}\right) d \tilde{x}_{k} \\
& f_{3}\left(\tilde{x}_{k}\right)=\int \Gamma_{1}\left(\widetilde{\zeta}_{k}, u_{k}\right) B^{T} N\left(\tilde{x}_{k} ; 0, I_{n}\right) d \tilde{x}_{k} \\
& f_{4}\left(\tilde{x}_{k}\right)=\int F_{1}\left(\widetilde{\zeta}_{k}, u_{k}\right) S \tilde{x}_{k}\left(F_{1}\left(\widetilde{\zeta}_{k}, u_{k}\right) S \widetilde{x}_{k}\right)^{T} \\
& \cdot N\left(\tilde{x}_{k} ; 0, I_{n}\right) d \tilde{x}_{k} \\
& f_{5}\left(\tilde{x}_{k}\right)=\int F_{1}\left(\widetilde{\zeta}_{k}, u_{k} S \tilde{x}_{k}\right) B^{T} N\left(\tilde{x}_{k} ; 0, I_{n}\right) d \tilde{x}_{k} .
\end{aligned}
$$

It is easy to prove that both $f_{1}\left(\tilde{x}_{k}\right)$ and $f_{3}\left(\tilde{x}_{k}\right)$ are the Gauss integrals for $\zeta_{k}$, since $\widehat{x}_{k}$ is constant vector, shown as

$$
\begin{aligned}
f_{1}\left(\tilde{x}_{k}\right) & =\int \Gamma_{1}\left(\widetilde{\zeta}_{k}, u_{k}\right) \Gamma_{1}^{T}\left(\widetilde{\zeta}_{k}, u_{k}\right) N\left(\widetilde{\zeta}_{k} ; 0, I_{l}\right) d \widetilde{\zeta}_{k} \\
& =\int \Gamma\left(\zeta_{k}, u_{k}\right) \Gamma^{T}\left(\zeta_{k}, u_{k}\right) N\left(\zeta_{k} ; \widehat{\zeta}, P_{\zeta, k}\right) d \zeta_{k} \\
f_{3}\left(\tilde{x}_{k}\right) & =\int \Gamma_{1}\left(\widetilde{\zeta}_{k}, u_{k}\right) B^{T} N\left(\widetilde{\zeta}_{k} ; 0, I_{l}\right) d \widetilde{\zeta}_{k} \\
& =\int \Gamma\left(\zeta_{k}, u_{k}\right) B^{T} N\left(\zeta_{k} ; \widehat{\zeta}, P_{\zeta, k}\right) d \zeta_{k} .
\end{aligned}
$$

Similar to the deduction for $E\left(F\left(\zeta_{k}, u_{k}\right) x_{k}\right)$, we have

$$
\begin{gathered}
f_{2}\left(\tilde{x}_{k}\right)=\int \Gamma_{1}\left(\widetilde{\zeta}_{k}, u_{k}\right)\left(\tilde{x}_{k}\right)^{T} S^{T} F_{1}^{T}\left(\widetilde{\zeta}_{k}, u_{k}\right) \\
\cdot N\left(\tilde{x}_{k} ; 0, I_{n}\right) d \tilde{x}_{k}=\int \Gamma\left(\zeta_{k}, u_{k}\right)
\end{gathered}
$$

$$
\begin{gathered}
\cdot\left[\left(S_{\zeta_{k}}\right)^{-1}\left(\zeta_{k}-\widehat{\zeta}_{k}\right)\right]^{T} S^{T} F_{2}^{T}\left(\zeta_{k}, u_{k}\right) \\
\cdot N\left(\zeta_{k} ; \widehat{\zeta}, P_{\zeta, k}\right) d \zeta_{k} \\
f_{5}\left(\widetilde{x}_{k}\right)=\int F_{1}\left(\widetilde{\zeta}_{k}, u_{k}\right) S \tilde{x}_{k} B^{T} N\left(\tilde{x}_{k} ; 0, I_{n}\right) d \tilde{x}_{k} \\
=\int F_{2}\left(\zeta_{k}, u_{k}\right) \\
\cdot S\left[\begin{array}{c}
\left(S_{\zeta_{k}}\right)^{-1}\left(\zeta_{k}-\widehat{\zeta}_{k}\right) \\
0
\end{array}\right] B^{T} N\left(\zeta_{k} ; \widehat{\zeta}, P_{\zeta, k}\right) d \zeta_{k} .
\end{gathered}
$$

So $f_{2}\left(\tilde{x}_{k}\right)$ and $f_{5}\left(\tilde{x}_{k}\right)$ are also the Gauss integrals for $\zeta_{k}$.

$$
\begin{aligned}
& f_{4}\left(\tilde{x}_{k}\right)=\int F_{1}\left(\widetilde{\zeta}_{k}, u_{k}\right) S \tilde{x}_{k}\left(\tilde{x}_{k}\right)^{T} S^{T} F_{1}^{T}\left(\widetilde{\zeta}_{k}, u_{k}\right) \\
& \cdot N\left(\tilde{x}_{k} ; 0, I_{n}\right) d \tilde{x}_{k}=\int F_{1}\left(\widetilde{\zeta}_{k}, u_{k}\right) \\
& \cdot S\left[\begin{array}{cc}
\tilde{\zeta}_{k}\left(\widetilde{\zeta}_{k}\right)^{T} & \widetilde{\zeta}_{k}\left(\widetilde{\eta}_{k}\right)^{T} \\
\tilde{\eta}_{k}\left(\widetilde{\zeta}_{k}\right)^{T} & \tilde{\eta}_{k}\left(\widetilde{\eta}_{k}\right)^{T}
\end{array}\right] S^{T} F_{1}^{T}\left(\widetilde{\zeta}_{k}, u_{k}\right) \\
& \cdot N\left(\tilde{x}_{k} ; 0, I_{n}\right) d \tilde{x}_{k} \text {. }
\end{aligned}
$$

Set

$$
\begin{aligned}
& \varphi\left(\widetilde{\zeta}_{k}\right)=F_{1}\left(\widetilde{\zeta}_{k}, u_{k}\right) \\
& \cdot S\left[\begin{array}{cc}
\tilde{\zeta}_{k}\left(\widetilde{\zeta}_{k}\right)^{T} & \tilde{\zeta}_{k}\left(\widetilde{\eta}_{k}\right)^{T} \\
\tilde{\eta}_{k}\left(\widetilde{\zeta}_{k}\right)^{T} & \widetilde{\eta}_{k}\left(\widetilde{\eta}_{k}\right)^{T}
\end{array}\right] S^{T} F_{1}^{T}\left(\widetilde{\zeta}_{k}, u_{k}\right) . \\
& \varphi\left(\widetilde{\zeta}_{k}\right)=\left[\left(\sum_{\alpha=1}^{l} \sum_{\beta=1}^{l} g_{\alpha, \beta}\left(\widetilde{\zeta}_{k}\right) \widetilde{\zeta}_{k, \alpha} \widetilde{\zeta}_{k, \beta}\right.\right. \\
& +2 \sum_{\alpha=1}^{l} \sum_{\beta=1}^{n-l} g_{\alpha, \beta+l}\left(\widetilde{\zeta}_{k}\right) \widetilde{\zeta}_{k, \alpha} \tilde{\eta}_{k, \beta} \\
& \left.\left.+\sum_{\alpha=1}^{n-l} \sum_{\beta=1}^{n-l} g_{\alpha+l, \beta+l}\left(\widetilde{\zeta}_{k}\right) \tilde{\eta}_{k, \alpha} \tilde{\eta}_{k, \beta}\right)_{i, j}\right]_{n \times n}
\end{aligned}
$$

According to Lemma 3, we have

$$
\begin{aligned}
& E\left(g_{\alpha, \beta+l}\left(\widetilde{\zeta}_{k}\right) \tilde{\zeta}_{k, \alpha} \tilde{\eta}_{k, \beta}\right)=0 \\
&(\alpha=1,2, \ldots, l ; \beta=1,2, \ldots, n-l) \\
& E\left(g_{\alpha+l, \beta+l}\left(\widetilde{\zeta}_{k}\right) \widetilde{\eta}_{k, \alpha} \widetilde{\eta}_{k, \beta}\right)=0 \\
&(\alpha=1,2, \ldots, n-l ; \beta=1,2, \ldots, n-l ; \alpha \neq \beta) \\
& E\left(g_{\alpha+l, \beta+l}\left(\widetilde{\zeta}_{k}\right) \widetilde{\eta}_{k, \alpha}^{2}\right)=E\left(g_{\alpha+l, \beta+l}\left(\widetilde{\zeta}_{k}\right)\right) \\
& \quad(\alpha=\beta=1,2, \ldots, n-l) .
\end{aligned}
$$


Hence,

$$
\begin{aligned}
E\left(\varphi\left(\widetilde{\zeta}_{k}\right)\right) & =\left[\left(\sum_{\alpha=1}^{l} \sum_{\beta=1}^{l} E\left(g_{\alpha, \beta}\left(\widetilde{\zeta}_{k}\right) \tilde{\zeta}_{k, \alpha} \tilde{\zeta}_{k, \beta}\right)+\sum_{\alpha=1}^{n-l} \sum_{\beta=1}^{n-l} E\left(g_{\alpha+l, \beta+l}\left(\widetilde{\zeta}_{k}\right)\right)\right)_{i, j}\right]_{n \times n} \\
& =\int F_{1}\left(\widetilde{\zeta}_{k}, u_{k}\right) S\left[\begin{array}{cc}
\widetilde{\zeta}_{k}\left(\widetilde{\zeta}_{k}\right)^{T} & 0 \\
0 & I_{n-l}
\end{array}\right] S^{T} F_{1}^{T}\left(\widetilde{\zeta}_{k}, u_{k}\right) N\left(\widetilde{\zeta}_{k} ; 0, I_{l}\right) d \widetilde{\zeta}_{k} \\
& =\int F_{2}\left(\zeta_{k}, u_{k}\right) S\left[\begin{array}{cc}
\left(S_{\zeta_{k}}\right)^{-1}\left(\zeta_{k}-\widetilde{\zeta}_{k}\right)\left(\zeta_{k}-\widetilde{\zeta}_{k}\right)^{T}\left(\left(S_{\zeta_{k}}\right)^{-1}\right)^{T} & 0 \\
0 & I_{n-l}
\end{array}\right] S^{T} F_{2}^{T}\left(\zeta_{k}, u_{k}\right) \cdot N\left(\zeta_{k} ; \widetilde{\zeta}_{k}, P_{\zeta, k}\right) d \zeta_{k}
\end{aligned}
$$

$f_{4}\left(\tilde{x}_{k}\right)$ is the Gauss integral for $\zeta_{k}$. It is proved that $P_{Z}$ is the Gauss integral for $\zeta_{k}$, shown as

$$
P_{Z}=\int \Psi^{*}\left(\zeta_{k}\right) N\left(\zeta_{k} ; \widehat{\zeta}_{k}, P_{\zeta, k}\right) d \zeta_{k}-\widehat{Z}(\widehat{Z})^{T}
$$

where

$$
\left.\begin{array}{l}
\left.\Psi\left(\zeta_{k}\right)=\Gamma\left(\zeta_{k}, u_{k}\right) \Gamma^{T}\left(\zeta_{k}, u_{k}\right)+\Gamma\left(\zeta_{k}, u_{k}\right)\left[\begin{array}{c}
\left.\left(S_{\zeta_{k}}\right)^{-1}\left(\zeta_{k}-\widehat{\zeta}_{k}\right)\right]^{T} \\
0
\end{array}\right]^{-1}\left(\zeta_{k}-\widehat{\zeta}_{k}\right)\right]^{T} \\
0
\end{array}\right] \begin{gathered}
\left(S_{\zeta_{k}}\right)^{T} F_{2}^{T}\left(\zeta_{k}, u_{k}\right)+\left(\Gamma\left(\zeta_{k}, u_{k}\right)\right. \\
\left.\cdot S^{T} F_{2}^{T}\left(\zeta_{k}, u_{k}\right)\right)^{T}+\Gamma\left(\zeta_{k}, u_{k}\right) B^{T}+\left(\Gamma\left(\zeta_{k}, u_{k}\right) B^{T}\right)^{T} \\
+F_{2}\left(\zeta_{k}, u_{k}\right) \\
\cdot S\left[\begin{array}{c}
\left(S_{\zeta_{k}}\right)^{-1}\left(\zeta_{k}-\tilde{\zeta}_{k}\right)\left(\zeta_{k}-\widetilde{\zeta}_{k}\right)^{T}\left(\left(S_{\zeta_{k}}\right)^{-1}\right)^{T} \\
0 \\
0
\end{array}\right] S^{T} F_{2}^{T}\left(\zeta_{k},\right. \\
\left.u_{k}\right)+F_{2}\left(\zeta_{k}, u_{k}\right) S\left[\begin{array}{c}
\left(S_{\zeta_{k}}\right)^{-1}\left(\zeta_{k}-\widehat{\zeta}_{k}\right) \\
0
\end{array}\right] B^{T}+\left(F_{2}\left(\zeta_{k}, u_{k}\right)\right. \\
\cdot S\left[\begin{array}{c}
\left.\left(S_{\zeta_{k}}\right)^{-1}\left(\zeta_{k}-\widehat{\zeta}_{k}\right) B_{B}^{T}\right)^{T}+B B^{T} . \\
0
\end{array}\right]
\end{gathered}
$$

Arrange (62) as

$$
\begin{aligned}
\Psi\left(\zeta_{k}\right)=\Phi & \left(\zeta_{k}\right)\left(\Phi\left(\zeta_{k}\right)\right)^{T}+\left(I-E_{k} H_{k+1} C_{k+1}\right) F\left(\zeta_{k}\right) \\
& \cdot S\left[\begin{array}{cc}
0 & 0 \\
0 & I_{n-l}
\end{array}\right] S^{T} F^{T}\left(\zeta_{k}\right) \\
& \cdot\left(I-E_{k} H_{k+1} C_{k+1}\right)^{T}
\end{aligned}
$$

Eq. (37) is proved.

By Definition 2, Theorem 4 gives the equivalent form of $\widehat{Z}$ (equivalent to $\widehat{x}_{k+1 / k}$ in (17)) and $P_{Z}$ (equivalent to $\widehat{P}_{x, k+1 / k}$ in (18)) when the reduced dimension SVDCKF is applied.
4.3. The Proposed NUIO. According to the Assumption 1 and NUIO in (28) to (29), $2 n$ sampling points are needed for the state in SVDCKF. As proved in Theorem $4, \widehat{P}_{x, k+1 / k}$ and $\widehat{x}_{k+1 / k}$ are the Gauss integrals for $\zeta_{k}$, shown as

$$
\begin{aligned}
\widehat{x}_{k+1 / k}= & \int \Phi\left(\zeta_{k}\right) N\left(\zeta_{k} ; \widehat{\zeta}_{k}, P_{\zeta, k}\right) d \zeta_{k} \\
\widehat{P}_{x, k+1 / k}= & \int \Psi\left(\zeta_{k}\right) N\left(\zeta_{k} ; \widehat{\zeta}_{k}, P_{\zeta, k}\right) d \zeta_{k} \\
& -\widehat{x}_{k+1 / k}\left(\widehat{x}_{k+1 / k}\right)^{T} .
\end{aligned}
$$

Therefore, when third-degree spherical-radial cubature rule is used to approximate the integrals in (64), only $2 l$ sampling points are needed.

Eqs. (13) and (14) are rewritten as

$$
\begin{aligned}
\widehat{P}_{\zeta_{k}} & =U_{\zeta, k}^{*}\left[\begin{array}{cc}
S_{\zeta, k}^{*} & 0 \\
0 & 0
\end{array}\right]\left(V_{\zeta, k}^{*}\right)^{T} \\
\widehat{X}_{i, k} & =U_{\zeta, k}^{*} \sqrt{S_{\zeta, k}^{*}} \xi_{i}+\widehat{\zeta}_{k} \quad i=1,2, \ldots, 2 l .
\end{aligned}
$$

Then (16), (17), and (18) are rewritten as

$$
\begin{aligned}
\widehat{X}_{i, k+1 / k} & =\Phi\left(\widehat{X}_{i, k}\right) \\
\widehat{x}_{k+1 / k} & =\frac{1}{2 l} \sum_{i=1}^{2 l} \widehat{X}_{i, k+1 / k} \\
\widehat{P}_{x, k+1 / k} & =\frac{1}{2 l} \sum_{i=1}^{2 l} \Psi\left(\widehat{X}_{i, k}\right)-\widehat{x}_{k+1 / k}\left(\widehat{x}_{k+1 / k}\right)^{T} .
\end{aligned}
$$

Note 3. Here, $S=U_{k} \sqrt{S_{k}^{*}}$ and $S_{\zeta_{k}}=U_{k} \sqrt{S_{\zeta, k}^{*}}$ in (38) and (39), respectively.

Replacing the corresponding equations by (65)-(66) in the algorithm given in Section 3, the NUIO based on SVDRDCKF is obtained and its flowchart is shown in Figure 1. In this algorithm, since the equivalent form of Theorem 4 is used in the propagation of nonlinear function, the third-order accuracy of the nonlinear function is guaranteed. 


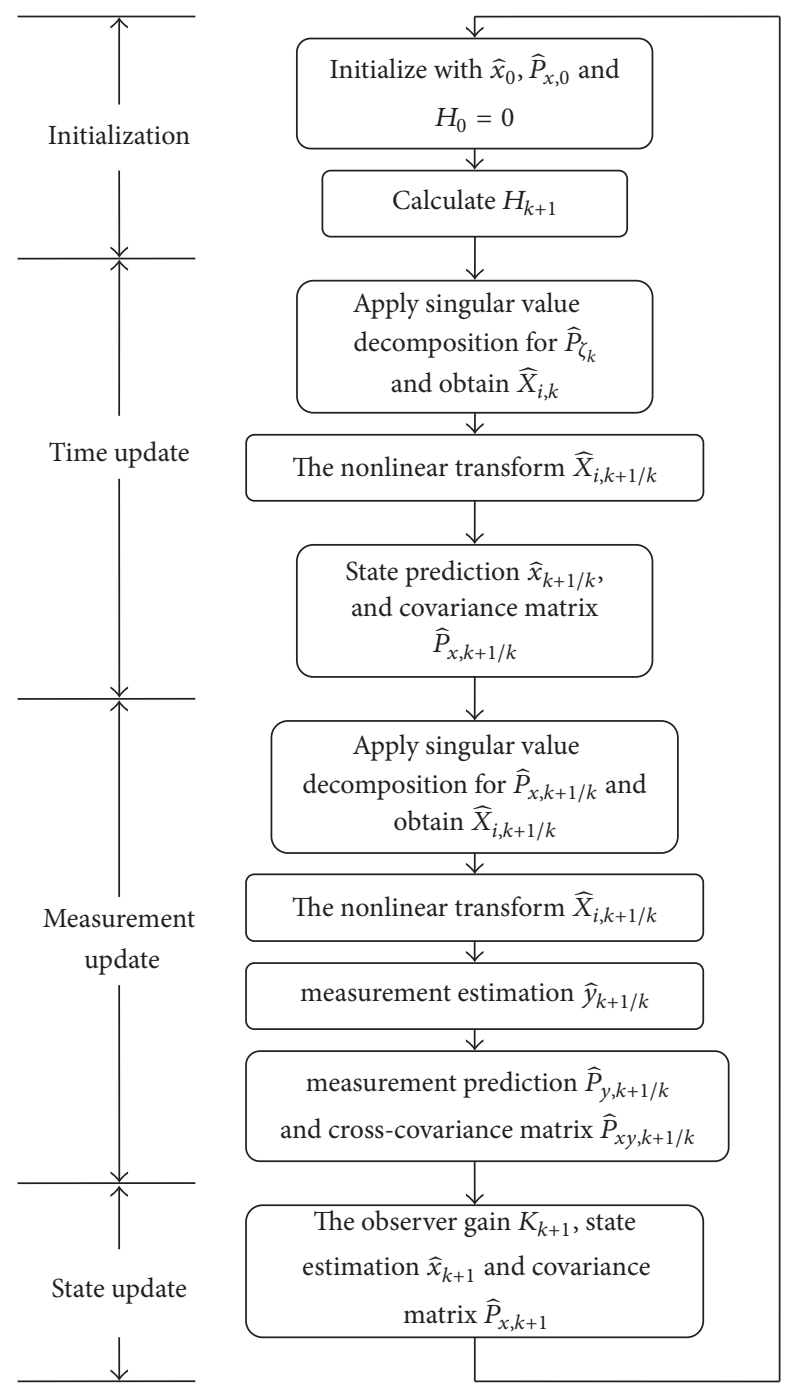

FIGURE 1: The flowchart of NUIO based on SVDRDCKF.

\section{Simulation and Analysis}

Consider the following nonlinear system, which is a maneuvering target tracking model with unknown inputs. It is necessary to estimate the position of the maneuvering target in the $X$ - and the $Y$-axis as well as their corresponding linear velocities.

$$
\begin{aligned}
& Z_{k+1}=\frac{Z_{k}}{2}+\frac{25 Z_{k}}{1+Z_{k}^{2}}+8 \cos (1.2(k-1))+r_{k} \\
& \chi_{k+1}=A \chi_{k}+B Z_{k+1}+E d_{k}+w_{k} \\
& y_{k+1}=D \chi_{k+1}+v_{k},
\end{aligned}
$$

where $\chi_{k+1}=\left[\begin{array}{llll}\chi_{1, k+1} & \chi_{2, k+1} & \chi_{3, k+1} & \chi_{4, k+1}\end{array}\right]^{T}$ is the position of the maneuvering target in the $X$ - and the $Y$-axis, as well as their corresponding linear velocities. $d_{k}=\left[\begin{array}{l}0.05 \sin (0.1 k \pi) \cos (0.1 k \pi) \\ 0.05 \sin (0.1 k \pi) \cos (0.1 k \pi)\end{array}\right]$ is the unknown input, which affects the linear velocity in the $X$ - and $Y$-axis of the maneuvering target. $Z_{k+1}$ is a scalar quantity as the maneuvering acceleration value of the target.

$$
\begin{aligned}
& A=\left[\begin{array}{cccc}
1 & 0 & 0.1 & 0 \\
0 & 1 & 0 & 0.1 \\
0 & 0 & 1 & 0 \\
0 & 0 & 0 & 1
\end{array}\right], \\
& B=\left[\begin{array}{cc}
1.25 \\
-1.25 \\
0.25 \\
-0.25
\end{array}\right], \\
& D=\left[\begin{array}{ccc}
1 & 0 & 0 \\
0 & 1 & 0
\end{array}\right], \\
& E=\left[\begin{array}{cc}
0 & 0 \\
0 & 0 \\
0.2 & 1 \\
1 & 0.2
\end{array}\right], \\
& w_{k} \sim N\left(0, Q_{w}\right) .
\end{aligned}
$$

Setting $x_{k}=\left[Z_{k}, \chi_{1, k}, \chi_{2, k}, \chi_{3, k}, \chi_{4, k}\right]^{T}=[X 1, X 2, X 3, X 4$, $X 5]^{T}$ and rewriting (67) into the form described by (26), we have

$$
\begin{aligned}
x_{k+1}= & {\left[\begin{array}{cc}
0.5 & 0 \\
0.5 B & A
\end{array}\right] x_{k}+g\left(Z_{k}\right) } \\
& +\left[\begin{array}{cc}
0 & 0 \\
0 & 0 \\
0 & 0 \\
0.2 & 1 \\
1 & 0.2
\end{array}\right]\left[\begin{array}{l}
0.05 \sin (0.1 k \pi) \cos (0.1 k \pi) \\
0.05 \sin (0.1 k \pi) \cos (0.1 k \pi)
\end{array}\right] \\
& +\left[\begin{array}{c}
r_{k} \\
B r_{k}+w_{k}
\end{array}\right] \\
y_{k+1}= & {\left[\begin{array}{ll}
0 & D
\end{array}\right] x_{k}+v_{k}, }
\end{aligned}
$$

where $g\left(Z_{k}\right)=\left[\begin{array}{l}1 \\ B\end{array}\right]\left(25 Z_{k} /\left(1+Z_{k}^{2}\right)+8 \cos (1.2(k-1))\right)$.

In order to justify the performances of the proposed algorithm, NUIO based on SVDRDCKF is applied for the estimation of $x_{k}$ compared with the NUIO based on SVDCKF. 


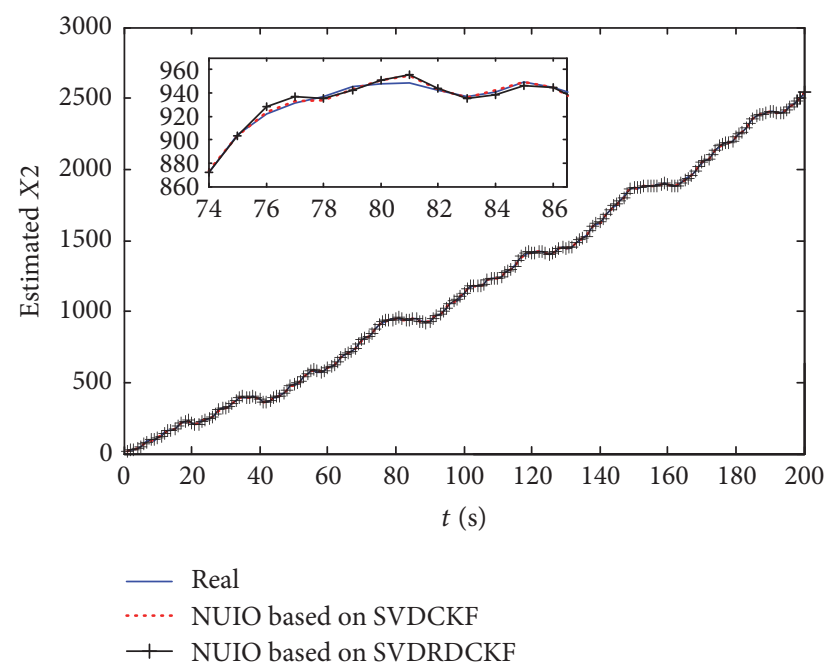

FIGURE 2: Estimated X2 applying NUIO based on SVDCKF and SVDRDCKF.

The initial value and related settings are given as follows:

$$
\begin{aligned}
\widehat{x}_{0} & =\left[\begin{array}{lllll}
0.1 & 20.1 & 30.1 & 1.3 & 1
\end{array}\right], \\
Q_{w} & =\left[\begin{array}{cccc}
0.09 & 0 & 0 & 0 \\
0 & 0.09 & 0 & 0 \\
0 & 0 & 0.09 & 0 \\
0 & 0 & 0 & 0.09
\end{array}\right], \\
Q_{v} & =\left[\begin{array}{llll}
9 & 0 & 0 & 0 \\
0 & 9 & 0 & 0 \\
0 & 0 & 9 & 0 \\
0 & 0 & 0 & 9
\end{array}\right], \\
P_{x, 0} & =\left[\begin{array}{lllll}
10 & 0 & 0 & 0 & 0 \\
0 & 10 & 0 & 0 & 0 \\
0 & 0 & 10 & 0 & 0 \\
0 & 0 & 0 & 1 & 0 \\
0 & 0 & 0 & 0 & 1
\end{array}\right] \\
Q_{r} & =2 .
\end{aligned}
$$

As proved in Theorem $4, \widehat{P}_{x, k+1 / k}$ and $\widehat{x}_{k+1 / k}$ are the Gauss integrals for the nonlinear part of the state $x_{k}$. For the system in (69), 10 sampling points should be selected according to NUIO based on SVDCKF. However, if the proposed reduced dimension algorithm is used, only 2 sampling points are needed for that there is only one element of $x_{k}$ causing the nonlinearity of the system. As the Gauss integral with 10 sampling points is equivalent to that with 2 points, NUIO based on SVDRDCKF algorithm in the estimation process still maintains the third-order accuracy for integral of the nonlinear state function, as well as that of SVDCKF.

Figures 2-5 present the estimation of position and the corresponding linear velocities in the $X$ - and $Y$-axis for

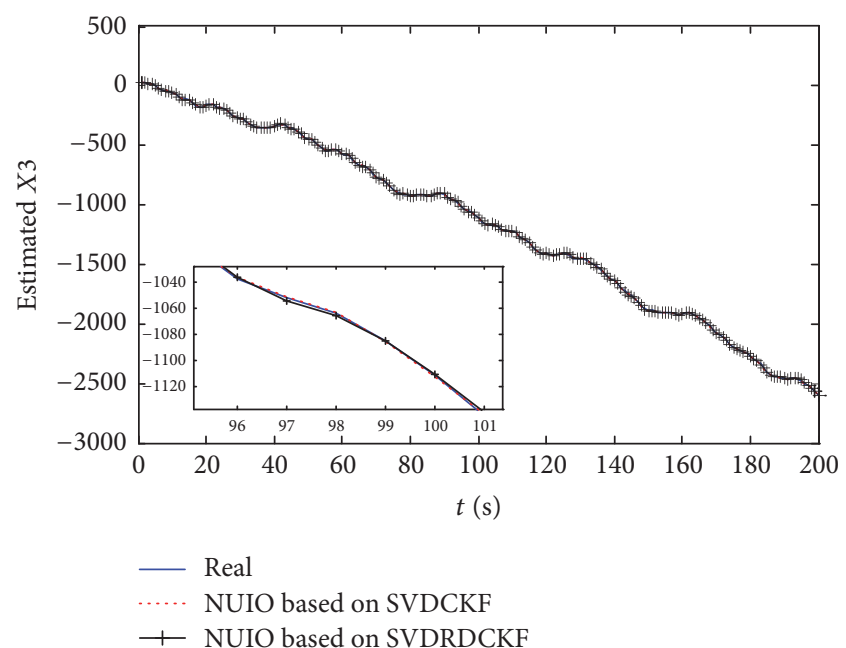

FIGURE 3: Estimated X3 applying NUIO based on SVDCKF and SVDRDCKF.

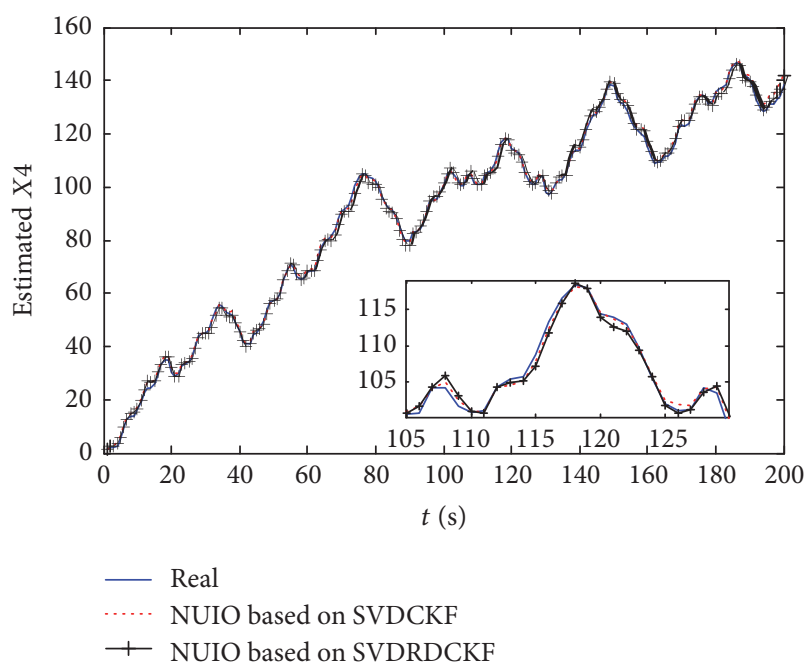

FIGURE 4: Estimated X4 applying NUIO based on SVDCKF and SVDRDCKF.

maneuvering target applying the NUIO based on SVDCKF and SVDRDCKF, respectively. As can be seen in these figures, the algorithm with 10 sampling points is very good to achieve the four state estimations of $x$. At the same time, we also see that, even if there are only 2 sampling points selected, the NUIO based on SVDRDCKF also achieves the estimation of the four states. Taking the subgraph in Figure 2 for example, the accuracy of the $X 2$ for the proposed algorithm is lower than the other, which is inevitable for the fewer sampling points.

This can also be verified by the relative errors (RE) of estimated position and linear velocities, shown in Figures 69. With the change of time, the values of the states estimated by the two algorithms are all close to the real values. This can be seen from the figures of RE, since their REs are eventually close to 0 . In the subgraph of Figure 9, it is obvious that the 


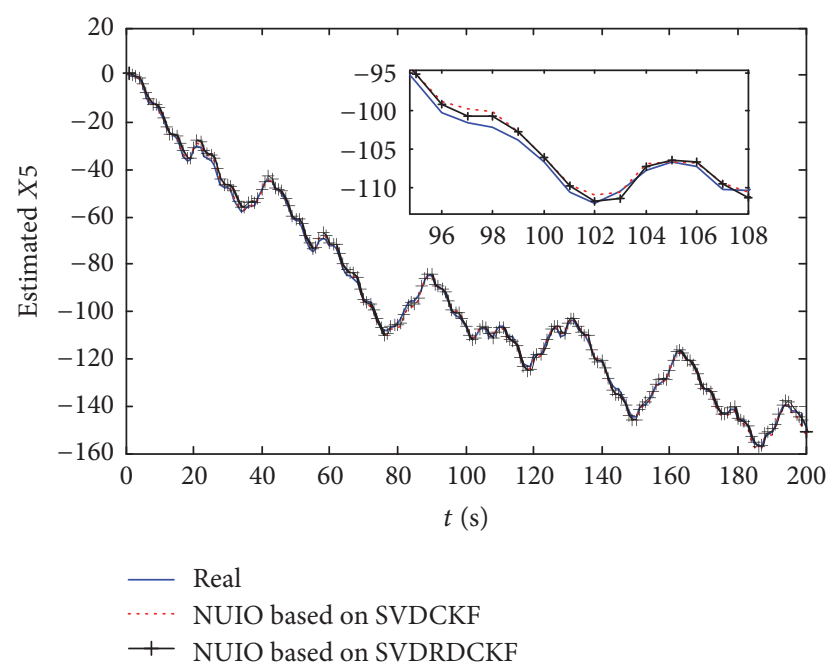

FIGURE 5: Estimated $X 5$ applying NUIO based on SVDCKF and SVDRDCKF.

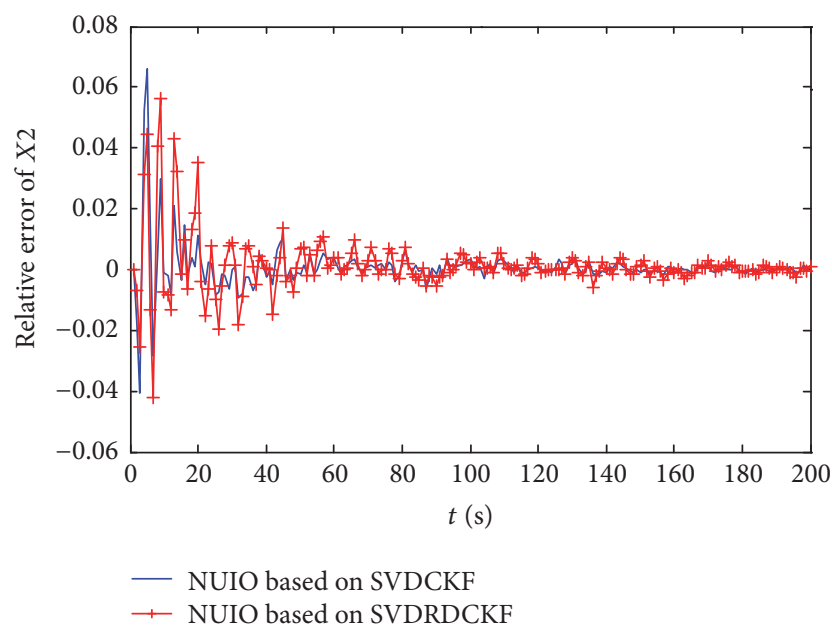

FIGURE 6: RE of $X 2$ applying NUIO based on SVDCKF and SVDRDCKF.

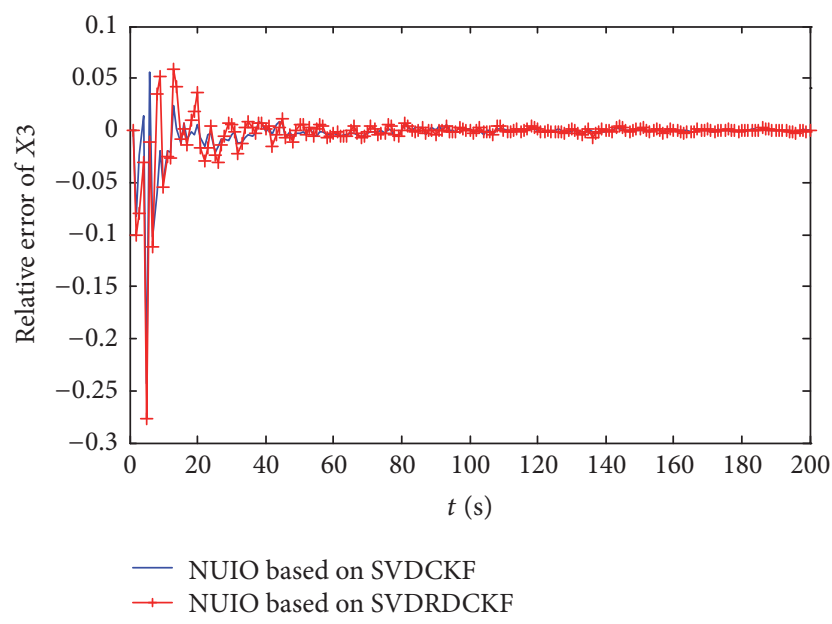

FIGURE 7: RE of $X 3$ applying NUIO based on SVDCKF and SVDRDCKF.

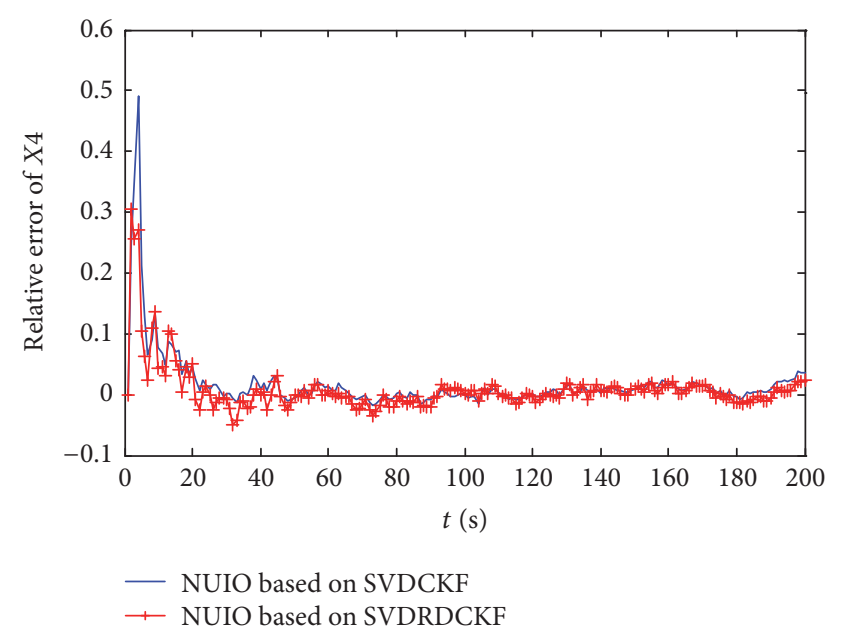

FIGURE 8: RE of $X 4$ applying NUIO based on SVDCKF and SVDRDCKF.

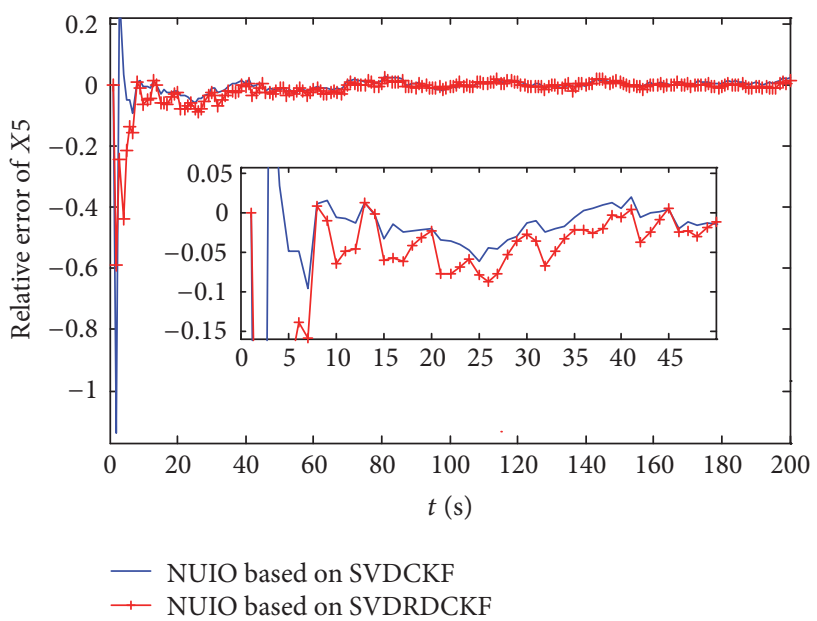

FIGURE 9: RE of $X 5$ applying NUIO based on SVDCKF and SVDRDCKF.

RE of NUIO based on SVDRDCKF is larger than that of SVDCKF.

To further compare the estimation performance of the two algorithms, 100 Monte-Carlo simulations are carried out, and the average absolute errors (MAD) of four states are shown in Figures 10-13, respectively.

The MAD of each state is larger by the proposed algorithm. But if the accuracies are permitted in applications, it can be seen that the MAD of the two algorithms is basically equivalent. Correspondingly, we greatly reduce the number of sampling points, reduced from 10 sampling points to only two. Hence, the NUIO based on SVDRDCKF can improve the calculating efficiency which can also maintain the thirdorder accuracy of Taylor's expansion.

In order to better demonstrate the excellent computational efficiency of proposed algorithm, we calculated the achieved average time cost of two algorithms across 100 Monte-Carlo experiments, shown in Table 1. The average time 


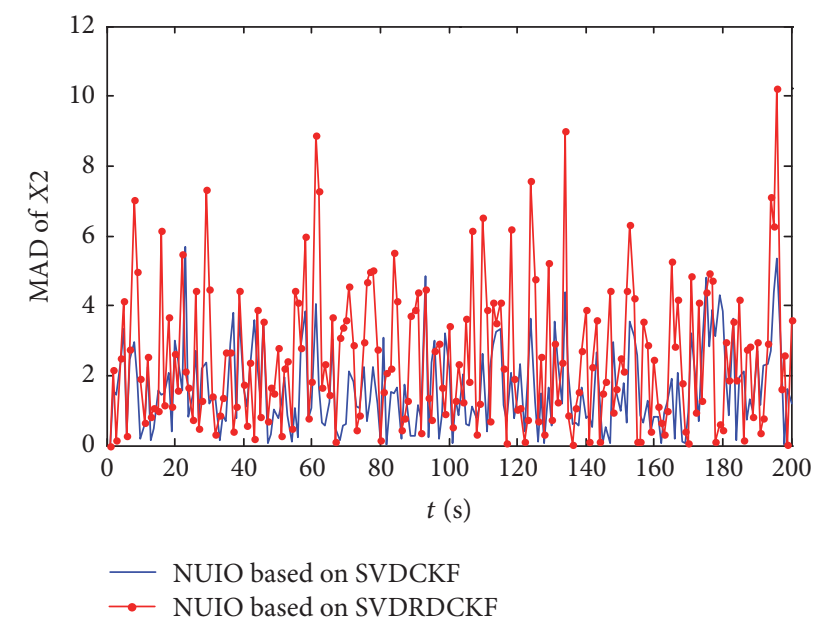

Figure 10: MAD of X2 applying NUIO based on SVDCKF and SVDRDCKF.

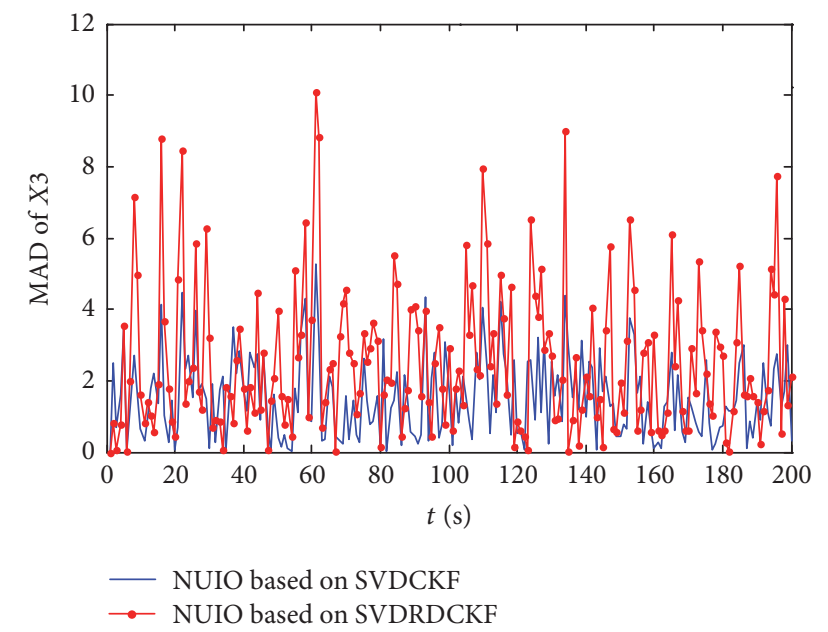

FIGURE 11: MAD of $X 3$ applying NUIO based on SVDCKF and SVDRDCKF.

TABLE 1: The average time of 100 Monte-Carlo simulations.

\begin{tabular}{ll}
\hline NUIO based on SVDCKF $(s)$ & 0.3246 \\
NUIO based on SVDRDCKF $(s)$ & 0.2656 \\
Saving time percent & $22.21 \%$ \\
\hline
\end{tabular}

of the proposed algorithm is $0.2656 \mathrm{~s}$ compared to 0.3246 of the SVDCKF. The proposed algorithm has saved $22.21 \%$ of the time. The result means that, although the accuracy is decreased by reducing sampling points, it is still in the acceptable range, and the computational efficiency has been significantly improved.

\section{Conclusion}

For some special nonlinear systems with unknown inputs, their nonlinearities are only caused by part of the state.

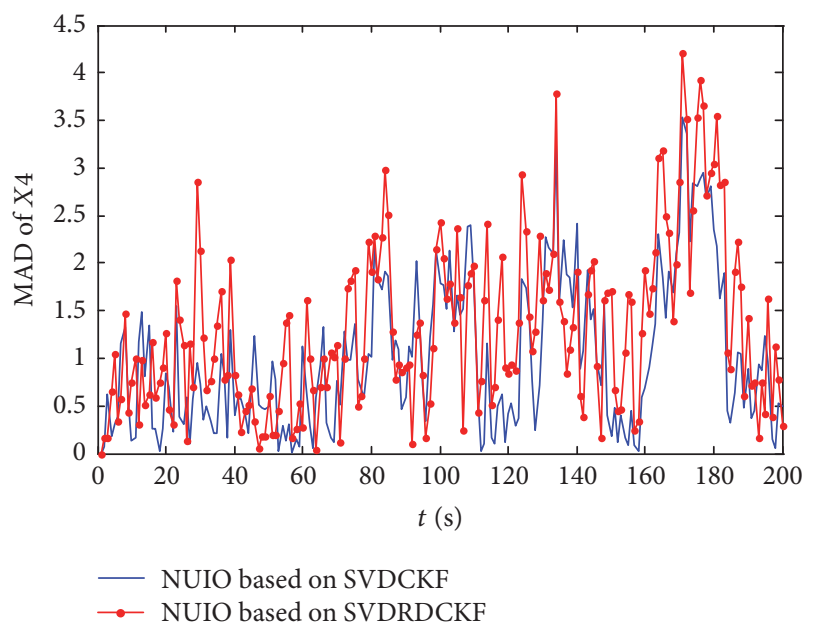

FIGURE 12: MAD of X4 applying NUIO based on SVDCKF and SVDRDCKF.

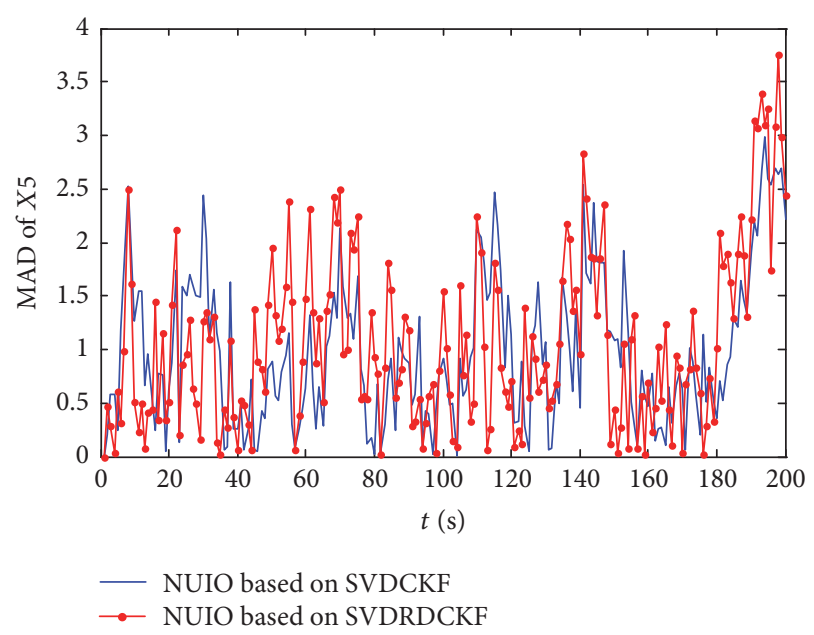

FIGURE 13: MAD of X5 applying NUIO based on SVDCKF and SVDRDCKF.

The paper gives a nonlinear unknown input observer based on singular value decomposition aided reduced dimension Cubature Kalman filter. The proposed algorithm maintains the third-order accuracy of Taylor's expansion for the integral of the nonlinear state function though sampling with the nonlinear part of the state instead of all. Simulation results show that the calculation efficiency is improved greatly while the filtering accuracy is guaranteed.

\section{Competing Interests}

The authors declare that they have no competing interests.

\section{References}

[1] A. Borboni and M. Lancini, "Commanded motion optimization to reduce residual vibration," Journal of Vibration \& Acoustics, vol. 137, no. 3, Article ID 031016, 9 pages, 2015. 
[2] R. E. Kalman, "A new approach to linear filtering and prediction problems," Journal of Basic Engineering, vol. 82, no. 1, pp. 35-45, 1960.

[3] A. H. Sayed, "A framework for state-space estimation with uncertain models," IEEE Transactions on Automatic Control, vol. 46, no. 7, pp. 998-1013, 2001.

[4] M. Fu, C. E. D. Souza, and L. Xie, " $H_{\infty}$ estimation for uncertain systems," International Journal of Robust and Nonlinear Control, vol. 2, no. 2, pp. 87-105, 1992.

[5] D. P. Bertsekas and I. B. Bertsekas, "Recursive state estimation for a set-membership description of uncertainty," IEEE Transactions on Automatic Control, vol. AC-16, pp. 117-128, 1971.

[6] Y. Theodor and U. Shaked, "Robust discrete-time minimumvariance filtering," IEEE Transactions on Signal Processing, vol. 44, no. 2, pp. 181-189, 1996.

[7] S. Sundaram and C. N. Hadjicostis, "Partial state observers for linear systems with unknown inputs," Automatica, vol. 44, no. 12, pp. 3126-3132, 2008.

[8] W. Fei, J. Xia, G. Ouyang, and J. Lin, "Sensor fault diagnosis for flight control system based on Cubature Kalman Filter," in Proceedings of the IEEE Chinese Guidance, Navigation and Control Conference, pp. 2657-2662, Yantai, China, August 2014.

[9] J. Zarei and J. Poshtan, "Design of nonlinear unknown input observer for process fault detection," Industrial and Engineering Chemistry Research, vol. 49, no. 22, pp. 11443-11452, 2010.

[10] B. D. O. Anderson, J. B. Moore, and M. Eslami, Optimal Filtering, Prentice-Hall, New Jersey, NJ, USA, 1979.

[11] E. A. Wan and R. Van Der Merwe, "The unscented Kalman filter for nonlinear estimation," in Proceedings of the IEEE Adaptive Systems for Signal Processing, Communications, and Control Symposium (AS-SPCC '00), pp. 153-158, IEEE, Alberta, Canada, October 2000.

[12] L. Nerger, W. Hiller, and J. Schröter, "A comparison of error subspace Kalman filters," Tellus Series A: Dynamic Meteorology and Oceanography, vol. 57, no. 5, pp. 715-735, 2005.

[13] Z.-L. Deng, Y. Gao, L. Mao, Y. Li, and G. Hao, "New approach to information fusion steady-state Kalman filtering," Automatica, vol. 41, no. 10, pp. 1695-1707, 2005.

[14] M. Gupta, L. Behera, V. K. Subramanian, and M. M. Jamshidi, "A robust visual human detection approach with UKF-based motion tracking for a mobile robot," IEEE Systems Journal, vol. 9, no. 4, pp. 1363-1375, 2015.

[15] W. Zhao, H. Li, L. Zou, and R. Yuan, "Adaptive and robust singular value decomposition aided cubature Kalman filter with chi-square test," Control and Intelligent Systems, vol. 44, no. 1, pp. 36-43, 2016.

[16] T. Yang, R. S. Laugesen, P. G. Mehta, and S. P. Meyn, "Multivariable feedback particle filter," Automatica, vol. 71, no. 1, pp. 10-23, 2016.

[17] D. Di Paola, A. Petitti, and A. Rizzo, "Distributed kalman filtering via node selection in heterogeneous sensor networks," International Journal of Systems Science, vol. 46, no. 14, pp. 25722583, 2015.

[18] D. Yu and S. Chakravorty, "A stochastic unknown input realization and filtering technique," Automatica, vol. 63, pp. 26-33, 2016.

[19] R. Yan, X. He, and D. H. Zhou, "Robust detection of intermittent faults for linear discrete-time stochastic systems with parametric perturbations," in Proceedings of the 34th Chinese Control Conference (CCC '15), pp. 6308-6313, IEEE, Hangzhou, China, July 2015.
[20] E. Rocha-Cózatl and J. A. Moreno, "Dissipative design of unknown input observers for systems with sector nonlinearities," International Journal of Robust \& Nonlinear Control, vol. 21, no. 14, pp. 1623-1644, 2011.

[21] H.-M. Qian, L. Ge, W. Huang, and Y. Peng, "Reduced dimension CKF algorithm and its application in SINS initial alignment," Systems Engineering and Electronics, vol. 35, no. 7, pp. 1492-1497, 2013.

[22] J. Chen and R. J. Patton, Robust Model-Based Fault Diagnosis for Dynamic Systems, Kluwer Academic Publishers, Dordrecht, the Netherlands, 1999. 


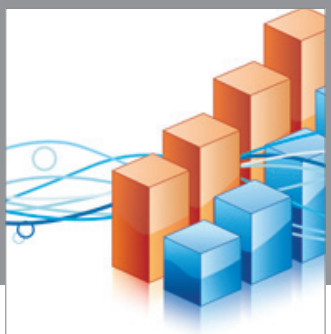

Advances in

Operations Research

vatem alat4

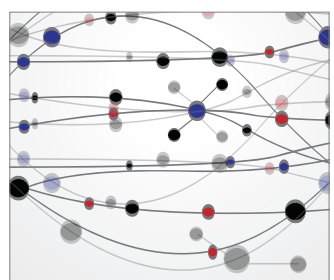

\section{The Scientific} World Journal
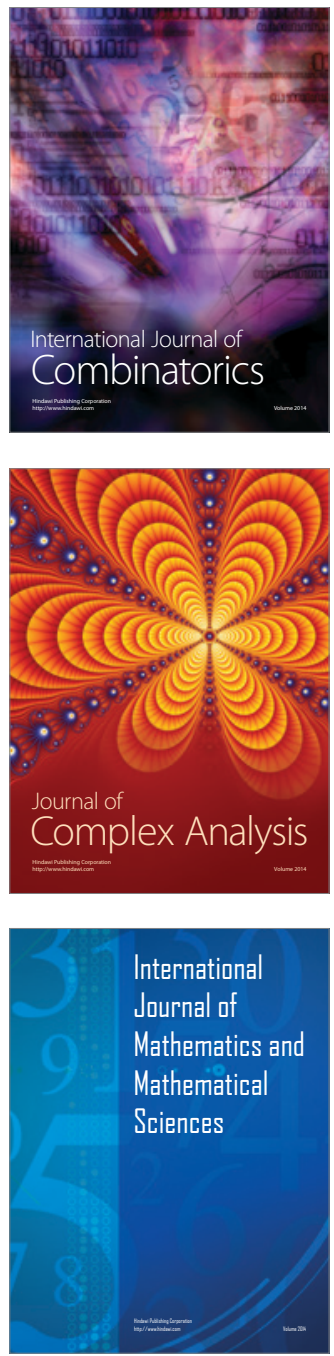
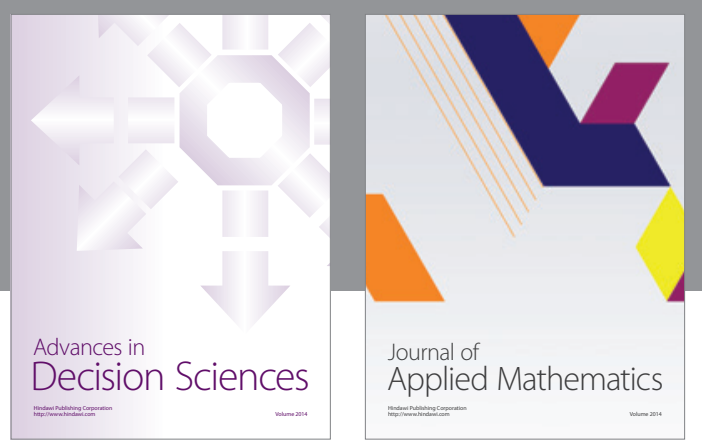

Algebra

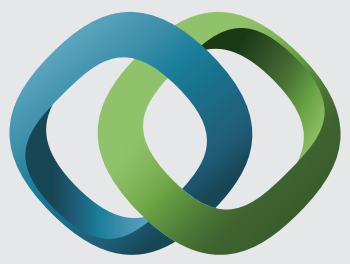

\section{Hindawi}

Submit your manuscripts at

https://www.hindawi.com
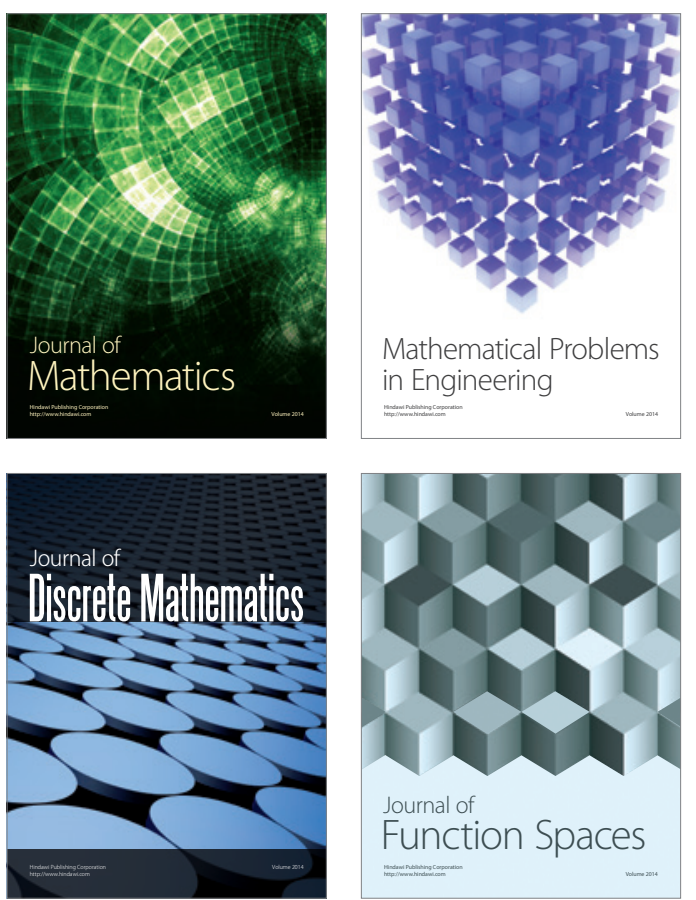

Mathematical Problems in Engineering
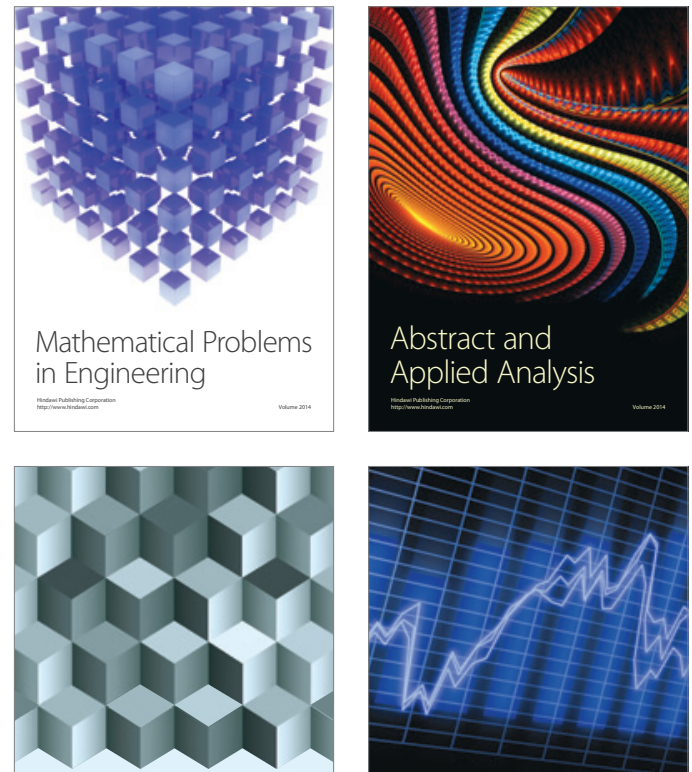

Journal of

Function Spaces

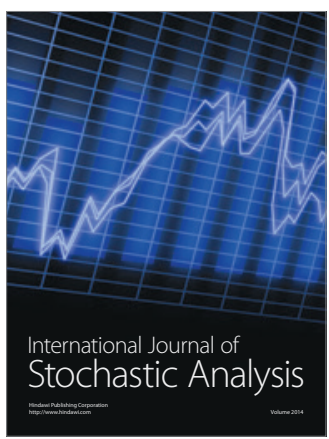

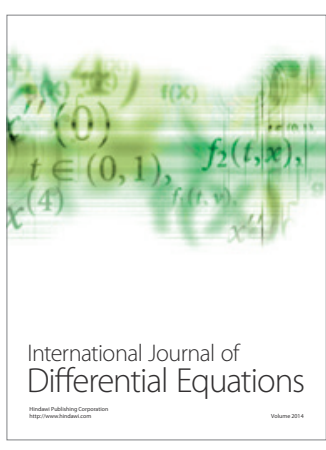
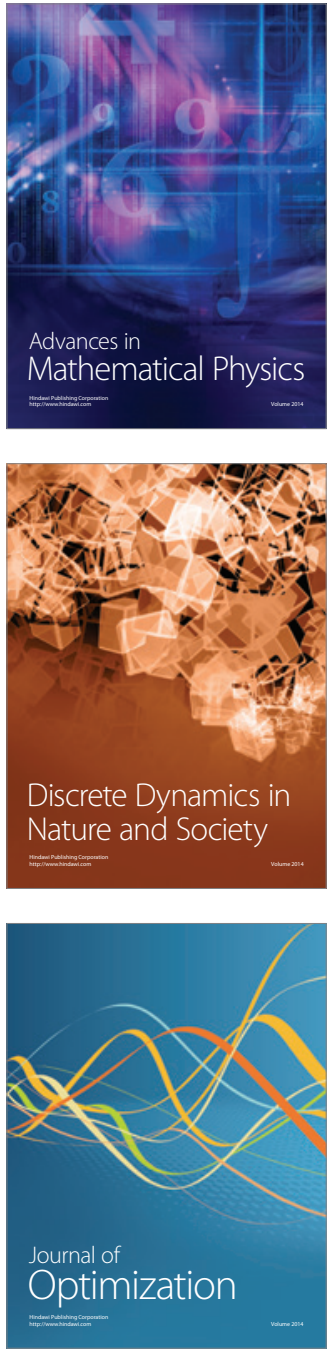